\title{
THE HERSCHEL AND JCMT GOULD BELT SURVEYS: CONSTRAINING DUST PROPERTIES IN THE PERSEUS B1 CLUMP WITH PACS, SPIRE, AND SCUBA-2
}

\author{
S. I. Sadavoy ${ }^{1,2}$, J. Di Francesco ${ }^{1,2}$, D. Johnstone ${ }^{1,2,3}$, M. J. Currie ${ }^{3}$, E. DrabeK ${ }^{4}$, J. Hatchell $^{4}$, D. Nutter ${ }^{5}$, Ph. André 6 , \\ D. Arzoumanian ${ }^{7}$, M. Benedettini ${ }^{8}$, J.-P. Bernard ${ }^{9,10}$, A. Duarte-Cabral ${ }^{11,12}$, C. Fallscheer $^{1,2}$, R. Friesen $^{13}$, \\ J. Greaves ${ }^{14}$, M. Hennemann ${ }^{6}$, T. Hill ${ }^{6}$, T. Jenness ${ }^{3}$, V. Könyves ${ }^{6,7}$, B. Matthews ${ }^{1,2}$, J. C. Mottram ${ }^{15}$, S. Pezzuto ${ }^{8}$,

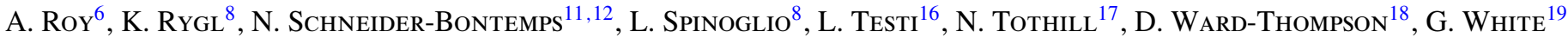 \\ AND \\ THE JCMT AND Herschel Gould Belt Survey teams \\ ${ }^{1}$ Department of Physics and Astronomy, University of Victoria, P.O. Box 355, STN CSC, Victoria, BC V8W 3P6, Canada \\ ${ }^{2}$ National Research Council Canada, 5071 West Saanich Road, Victoria, BC V9E 2E7, Canada \\ ${ }^{3}$ Joint Astronomy Centre, 660 N. A'ohōkū Place, University Park, Hilo, HI 96720, USA \\ ${ }^{4}$ School of Physics, University of Exeter, Stocker Road, Exeter EX4 4QL, UK \\ ${ }^{5}$ School of Physics and Astronomy, Cardiff University, Queen's Buildings, The Parade, Cardiff CF24 3AA, UK \\ ${ }^{6}$ Laboratoire AIM, CEA/DSM-CNRS-Université Paris Diderot, IRFU/Service d'Astrophysique, Saclay, F-91191 Gif-sur-Yvette, France \\ ${ }^{7}$ IAS, CNRS (UMR 8617), Université Paris-Sud 11, Bâtiment 121, F-91400 Orsay, France \\ ${ }^{8}$ Istituto di Astrofisica e Planetologia Spaziali, via Fosso del Cavaliere 100, I-00133 Rome, Italy \\ ${ }^{9}$ CNRS, IRAP, 9 Av. colonel Roche, BP 44346, F-31028 Toulouse Cedex 4, France \\ ${ }^{10}$ Université de Toulouse, UPS-OMP, IRAP, F-31028 Toulouse Cedex 4, France \\ ${ }^{11}$ Université de Bordeaux, LAB, UMR 5804, F-33270 Floirac, France \\ ${ }^{12}$ CNRS, LAB, UMR 5804, F-33270 Floirac, France \\ ${ }^{13}$ Dunlap Institute, University of Toronto, Toronto, ON M5S 3H8, Canada \\ ${ }^{14}$ School of Physics and Astronomy, University of St. Andrews, North Haugh, St. Andrews, Fife KY16 9SS, UK \\ ${ }^{15}$ Leiden University, 506 Huygens Laboratory, P.O. Box 9513, 2300-RA Leiden, The Netherlands \\ ${ }^{16}$ ESO, Karl Schwarzschild-Strasse 2, D-85748 Garching bei München, Germany \\ 17 University of Western Sydney, Locked Bag 1797, Penrith, NSW 2751, Australia \\ 18 Jeremiah Horrocks Institute, University of Central Lancashire, Preston, Lancashire PR1 2HE, UK \\ ${ }^{19}$ Department of Physics and Astronomy, The Open University, Milton Keynes MK7 6AA, UK \\ Received 2012 December 7; accepted 2013 March 6; published 2013 April 4
}

\begin{abstract}
We present Herschel observations from the Herschel Gould Belt Survey and SCUBA-2 science verification observations from the JCMT Gould Belt Survey of the B1 clump in the Perseus molecular cloud. We determined the dust emissivity index using four different techniques to combine the Herschel PACS+SPIRE data at 160-500 $\mu \mathrm{m}$ with the SCUBA-2 data at $450 \mu \mathrm{m}$ and $850 \mu \mathrm{m}$. Of our four techniques, we found that the most robust method was filtering out the large-scale emission in the Herschel bands to match the spatial scales recovered by the SCUBA-2 reduction pipeline. Using this method, we find $\beta \approx 2$ toward the filament region and moderately dense material and lower $\beta$ values $(\beta \gtrsim 1.6$ ) toward the dense protostellar cores, possibly due to dust grain growth. We find that $\beta$ and temperature are more robust with the inclusion of the SCUBA-2 data, improving estimates from Herschel data alone by factors of $\sim 2$ for $\beta$ and by $\sim 40 \%$ for temperature. Furthermore, we find core mass differences of $\lesssim 30 \%$ compared to Herschel-only estimates with an adopted $\beta=2$, highlighting the necessity of long-wavelength submillimeter data for deriving accurate masses of prestellar and protostellar cores.
\end{abstract}

Key words: dust, extinction - ISM: clouds - stars: formation - stars: low-mass - stars: protostars

Online-only material: color figures

\section{INTRODUCTION}

Molecular clouds are composed of dust and molecular gas ranging from $\sim 10^{2} \mathrm{~cm}^{-3}$ for the large-scale, low-density cloud to $>10^{4} \mathrm{~cm}^{-3}$ for the small-scale, dense star-forming "cores" (Bergin \& Tafalla 2007). These molecular clouds are often traced by their cold dust emission, which peak at far-infrared or submillimeter wavelengths, and many studies have used optically thin dust emission to measure masses and column densities of cores and filaments (e.g., Kirk et al. 2006; Enoch et al. 2009; André et al. 2010; Arzoumanian et al. 2011). A key parameter to convert dust emission into mass (or column density) is the dust opacity, which corresponds to the ability of dust grains to absorb radiation. Unfortunately, the dust opacity is one of the most difficult observational measurements, and uncertainties in the dust opacity can result in significant mass uncertainties (Henning et al. 1995; Shirley et al. 2011; Martin et al. 2012). Since dust populations in molecular clouds are expected to change due to dust coagulation and the formation of icy mantles in the densest environments (Draine \& Lee 1984; Ossenkopf \& Henning 1994), it is important to determine the dust opacity for both the large-scale cloud and the small-scale cores.

Generally, submillimeter dust opacities assume a power-law form such that $\kappa_{v} \sim \nu^{\beta}$, where $\beta$ is the dust emissivity index. Since the spectral energy distribution (SED) of cold, dusty starforming regions are best represented by graybody emission, $\beta$ is critical to the SED fit. Several studies have attempted to determine $\beta$ toward specific prestellar or protostellar cores using long-wavelength submillimeter emission (e.g., Friesen et al. 2005; Schnee et al. 2010; Shirley et al. 2011). These studies, however, were limited in wavelength coverage and poor map sensitivities. Conversely, recent observations with the Herschel Space Observatory (Pilbratt et al. 2010) extend our ability to measure cold dust emission to larger scales over multiple wavelengths. In particular, the Photodetector Array 
Camera and Spectrometer (PACS; Poglitsch et al. 2010) and the Spectral and Photometric Imaging Receiver (SPIRE; Griffin et al. 2010) have provided unprecedented sensitivity for farinfrared and submillimeter wavelengths at 70-500 $\mu \mathrm{m}$, which cover the SED peak from cold dust at $T \sim 10-40 \mathrm{~K}$. Both $\beta$ and temperature, however, affect the curvature of the blackbody function, and thus are degenerate toward the SED peak. To break this degeneracy, $\beta$ can be constrained from the Rayleigh-Jeans tail of the SED (Doty \& Leung 1994; Shetty et al. 2009b), i.e., $\lambda \gg 300 \mu \mathrm{m}$ for cold star-forming regions at $T \lesssim 30 \mathrm{~K}$. Therefore, additional long-wavelength data at good resolution and sensitivity are necessary to constrain the dust emissivity.

These requirements make the Submillimetre Common-User Bolometer Array-2 (SCUBA-2; Holland et al. 2013) at the James Clerk Maxwell Telescope (JCMT) an excellent complement to PACS and SPIRE data. SCUBA-2 can simultaneously observe at $450 \mu \mathrm{m}$ and $850 \mu \mathrm{m}$, both at higher resolutions than the three SPIRE bands (the JCMT primary mirror is five times larger than Herschel's). Therefore, SCUBA-2 observations provide necessary data along the Rayleigh-Jeans tail and excellent spatial resolution at longer wavelengths.

To explore possible variations of $\beta$ toward cold cores, we have combined PACS+SPIRE data with SCUBA-2 data from the JCMT Gould Belt Survey (GBS) for the first time. For this study, we have selected the B1 clump of the Perseus molecular cloud. B1 was previously observed with Herschel as part of the Herschel GBS (Sadavoy et al. 2012; Pezzuto et al. 2012), and was the first field of the JCMT GBS (Ward-Thompson et al. 2007) that nominally reached the SCUBA-2 survey depth. B1 is a nearby star-forming region at $235 \mathrm{pc}$ (Hirota et al. 2008) with a total clump mass of $\sim 1200 M_{\odot}$ and $\sim 10$ dense submillimeter cores grouped into a central cluster (Kirk et al. 2006). B1 is also relatively young (compared with the nearby NGC 1333 and IC 348 clumps), containing a high percentage $(>40 \%)$ of Class I young stellar objects, of which several drive outflows (Jørgensen et al. 2008; Bally et al. 2008). Furthermore, a recent study by Pezzuto et al. (2012) suggests that two dense cores in B1 are excellent candidates for first hydrostatic cores based on Herschel detections at $\gtrsim 100 \mu \mathrm{m}$ with no corresponding Spitzer detections at $24 \mu \mathrm{m}$. Additionally, Herschel observations of B1 revealed several pillar-like structures (Sadavoy et al. 2012), suggesting that a strong ionization front may be influencing the clump. This front likely originates from 40 Per, a high-mass main-sequence star in the Perseus OB2 region (see Bally et al. 2008).

This paper is organized as follows. In Section 2, we describe our Herschel and SCUBA-2 data sets, as well as CO (3-2) observations of B1. In Section 3, we show our results, first from our SCUBA-2 continuum and CO (3-2) observations, and then from fitting SEDs to the Herschel-only bands. In Section 4, we determine $\beta$ using the PACS+SPIRE and SCUBA-2 bands, following four different techniques to combine these data. In Section 5, we use our most robust technique to probe the variations in dust opacity and core mass, and we discuss the implications of our analyses for future studies that will combine SCUBA-2 and PACS+SPIRE data. In Section 6, we summarize our results.

\section{DATA}

\subsection{Herschel Observations}

The western half of Perseus, including the B1 clump, was observed with Herschel in 2010 February as part of the Herschel GBS. These data consist of parallel photometric observations with the PACS and SPIRE instruments at $70 \mu \mathrm{m}, 160 \mu \mathrm{m}$, $250 \mu \mathrm{m}, 350 \mu \mathrm{m}$, and $500 \mu \mathrm{m}$ at a $60 \operatorname{arcsec~s}^{-1}$ scan rate. The PACS and SPIRE raw data were reduced with version 7.0 of HIPE using modified reduction scripts by M. Sauvage (PACS) and P. Panuzzo (SPIRE) and updated calibration corrections; we used the PACS Calibration Set ver. 26 and the SPIRE Calibration Tree 6.1 for our PACS and SPIRE reductions, respectively. The final maps were produced using version 11 of the scanamorphos routine (Roussel 2012). For more information, see Sadavoy et al. (2012). For more information about the observations of Perseus and an alternative map making routine, see Pezzuto et al. (2012). The Herschel observations have resolutions of $\sim 8^{\prime \prime}-36^{\prime \prime}$.

\subsection{SCUBA-2 Observations}

B1 was observed at $450 \mu \mathrm{m}$ and $850 \mu \mathrm{m}$ with SCUBA-2 as part of Science Verification (hereafter S2SV) observations for the JCMT GBS in 2011 October. SCUBA-2 is a bolometer detector with $\sim 10,000$ pixels spread over eight science arrays for a field of view of $\sim 45 \operatorname{arcmin}^{2}$ (Holland et al. 2013). For comparison, the predecessor bolometer detector, SCUBA, contained only 128 pixels over two science arrays for a field of view of $\sim 5 \operatorname{arcmin}^{2}$ (Holland et al. 1999). For S2SV, all eight science grade arrays (four arrays for each band) were available.

The B1 clump was observed six times on October 18 in very dry (Grade $1 ; \tau_{225}<0.05$ ) weather and three more times on October 19 in poorer conditions (Grade 3; $\tau_{225}<0.1$ ). Each observation used a PONG1800 observing pattern (Dempsey et al. 2012; Holland et al. 2013), which involved mapping a $38^{\prime} \times 38^{\prime}$ square box centered at $3^{\mathrm{h}} 33^{\mathrm{m}} 12^{\mathrm{s}} .7,+31^{\circ} 06^{\prime} 48^{\prime \prime}(\mathrm{J} 2000)$ with a mapping speed of $480 \operatorname{arcsec~} \mathrm{s}^{-1}$ and scan spacing of $30^{\prime \prime}$. The box was mapped five times, rotating the sides by $18^{\circ}$ with respect to the sky between each coverage to produce a well-sampled circular map of $\sim 30^{\prime}$ diameter. This pattern was repeated to increase the sensitivity of the map. Two of the six observations on October 18 were made at high elevations $\left(>65^{\circ}\right)$, which caused erratic telescope tracking motions during the PONG-mode mapping and introduced corrugated artifacts into the respective images.

The S2SV observations were reduced using the Starlink SMURF version 1.4.0 package (Jenness et al. 2011; Chapin et al. 2013) and the makemap routine, which generates a map of the sky by iteratively fitting a number of different signals in the data. These signals included the common mode signal (caused by atmospheric emission and oscillations in the refrigerator system), the gain of each bolometer, the atmospheric extinction, and the astronomical signal. The mapmaker iterated until a convergence parameter was reached. ${ }^{20}$ Each observation was reduced independently in this manner and then an initial coadded map was produced from the independent observations. We combined the four good observations in Grade 1 weather, rejecting the three Grade 3 observations and two corrugated Grade 1 observations.

An initial co-added map was used to generate a mask that encompassed all parts of the map that contain significant levels of flux. This mask was used to re-reduce all of the data in the manner described above, except the astronomical signal is

\footnotetext{
20 The convergence parameter was defined as $\bar{C}_{i}=$ $\left(1 / N_{i}\right) \sum\left|F_{i, p}-F_{i-1, p}\right| / \sqrt{\sigma_{i, p}}$, where $i$ is the $i$ th iteration, $p$ corresponds to each pixel within the current mask, $N_{i}$ is the total number of pixels within the mask, $F_{i, p}$ is the flux in each pixel for the $i$ th iteration of the map, and $\sigma_{i, p}$ is the variance in each pixel for the $i$ th iteration. The convergence parameter was measured as an average over all pixels. The $i$ th iteration converged when $\bar{C}_{i} \leqslant 0.001$.
} 

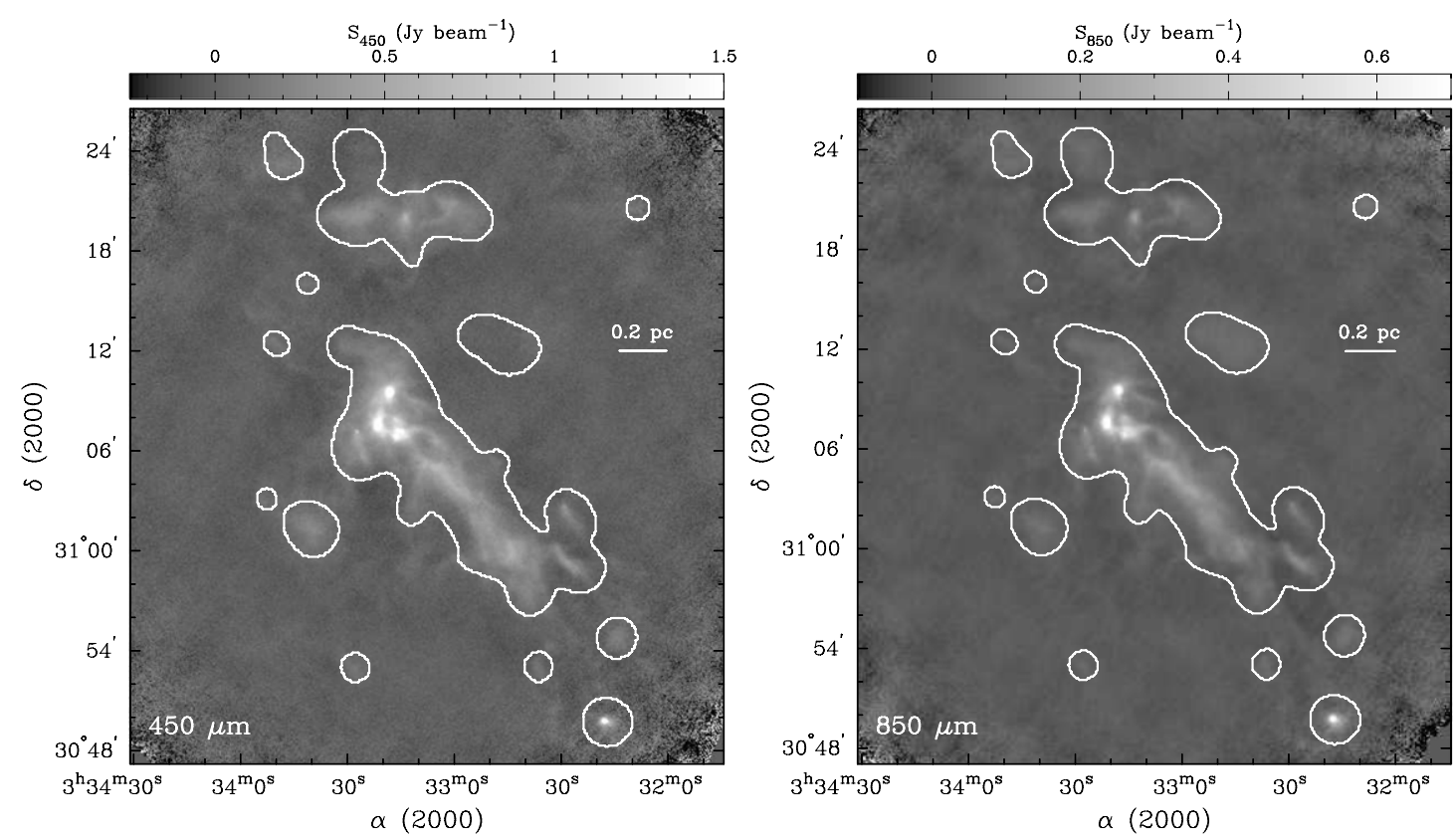

Figure 1. SCUBA-2 observations of the B1 clump at $450 \mu \mathrm{m}$ (left) and $850 \mu \mathrm{m}$ (right). Contours show the masked regions used in the reductions. Areas outside of the mask have uncertain fluxes.

only retained between each iteration if it lies within the masked area. The purpose of using the mask in this manner was to prevent spurious artifacts in the low-sensitivity regions of the map. For simplicity, the mask was generated corresponding to those regions with signal-to-noise ratios $\gtrsim 5$ at $850 \mu \mathrm{m}$. Regions outside the mask, however, were not used in any analysis, as the data there did not iterate to convergence. We tested the robustness of the maps by inserting artificial sources into the raw data and recovering reliable fluxes in the final map. Figure 1 shows the final reduced maps, highlighting the masked regions. The same mask was used for the $450 \mu \mathrm{m}$ reductions.

We used flux conversion factor (FCF) values of $491 \mathrm{Jy} \mathrm{beam}^{-1} \mathrm{pW}^{-1}$ and $556 \mathrm{Jy}$ beam $^{-1} \mathrm{pW}^{-1}$ for the $450 \mu \mathrm{m}$ and $850 \mu \mathrm{m}$ maps, respectively, based on observations of standard calibration sources (Dempsey et al. 2012, 2013). These FCF values corresponded to the version of Starlink used in our reduction. We adopted a calibration uncertainty of $10 \%$ across the SCUBA-2 $850 \mu \mathrm{m}$ map (Dempsey et al. 2012). For the $450 \mu \mathrm{m}$ band, however, atmospheric variability becomes more significant, even in Grade 1 weather. Therefore, we adopted a $50 \%$ calibration uncertainty at $450 \mu \mathrm{m}$, which was the typical flux error at $450 \mu \mathrm{m}$ adopted for the SCUBA Legacy Survey (Di Francesco et al. 2008). We acknowledge that such a large calibration error is conservative and with further investigation, will be improved. The final $1 \sigma$ rms sensitivity of the observed SCUBA-2 map was $\sim 7 \mathrm{mJy}^{\text {beam }}{ }^{-1}$ at $850 \mu \mathrm{m}$ over a $6^{\prime \prime}$ pixel grid. The effective $1 \sigma$ point-source sensitivity integrated over the beam area was $\sim 3 \mathrm{mJy}$, the target set by the SCUBA-2 GBS (Ward-Thompson et al. 2007). For the $450 \mu \mathrm{m}$ map, the final $1 \sigma$ rms sensitivity was $\sim 28 \mathrm{mJy}^{\text {beam }}{ }^{-1}$ over a $4^{\prime \prime}$ pixel grid for an equivalent point-source sensitivity of $\sim 11 \mathrm{mJy}$ over the beam area.

The SCUBA-2 beam contains two components, the primary beam and an error beam (e.g., Di Francesco et al. 2008). The $450 \mu \mathrm{m}$ and $850 \mu \mathrm{m}$ primary beams are $\sim 8^{\prime \prime}$ and $\sim 13^{\prime \prime}$, and the error beams are $\sim 25^{\prime \prime}$ and $\sim 48^{\prime \prime}$, respectively. At $450 \mu \mathrm{m}$, the error beam accounted for $\sim 40 \%$ of the volume, whereas at $850 \mu \mathrm{m}$, the error beam accounted for $\sim 25 \%$ of the volume (Dempsey et al. 2013; see also Section 3.1). For this paper, we adopted effective Gaussian beams with FWHM values of 9.3 and $14^{\prime \prime} .2$ for $450 \mu \mathrm{m}$ and $850 \mu \mathrm{m}$, respectively.

\subsection{HARP Observations}

We mapped the B1 clump in $\mathrm{CO}(3-2)$ line emission with the Heterodyne Array Receiver Program (HARP; see Buckle et al. 2009 and references therein) on 2012 June 29 in good Grade 2 weather $\left(\tau_{225}<0.08\right)$ over three hours. Briefly, the HARP instrument consists of 16 heterodyne detectors arranged in a $4 \times 4$ pixel grid and covering a frequency range of $325-375 \mathrm{GHz}$. Observations from HARP are processed by the Auto-Correlation Spectral Imaging System (ACSIS; Jenness et al. 2008). For these observations, only 14 receptors were functional. We made two position-switch raster maps (in orthogonal directions) over a $30^{\prime} \times 30^{\prime}$ region coincident with the S2SV-mapped region. The backend was configured to a $1 \mathrm{GHz}$ bandwidth over 2048 channels for a velocity resolution of $\sim 0.42 \mathrm{~km} \mathrm{~s}^{-1}$.

The data were reduced using the ORAC-DR (Cavanagh et al. 2008) pipeline for ACSIS, and the reduction recipe outlined below can be found in the latest Starlink release. Heterodyne detectors are notorious for noisy baselines due to external interferences. Rather than reject the entire spectra from these detectors, we performed an iterative reduction to remove suspect spectra from the time series while keeping the good spectra, thereby improving the signal to noise and the coverage of the final spectral map. The reduction pipeline can be broadly divided into two parts.

First, we performed an initial processing of each observation, which includes chronological sorting of the raw time-series cube and a quality-assurance stage. Of particular relevance to the B1 data was the removal of spectra affected by high- and low-frequency interferences. To identify these interferences, we trimmed the excessively noisy ends of the spectra and excluded the spectral region where the astronomical signal was present. High-frequency noise (three to four spectral elements) was detected using a one-dimensional Laplacian filter along the spectral axis for each receptor, and the affected blocks of spectra were identified by a threshold rms profile along the time axis. 
Low-frequency oscillations (or distortions in the baseline) were found by smoothing features smaller than 100 pixels, and we summed the rms deviations from the best-linear baseline fit to measure the nonlinearity for each spectrum. Again, we used a threshold to identify spectra that contained low-frequency artifacts. Spectra or whole receptors that failed quality assurance were rejected.

Second, we applied an iterative procedure, which refined the baseline subtraction at each cycle. We combined and regridded all the raw time-series cubes into a group spatial cube. Then, we applied a baseline subtraction and smoothing so that the astronomical emission was located within the group cube using clump finding. The emission defines a further mask, which is converted back to a time series and is applied to the raw timeseries cube for the next iteration. In practice, one iteration was sufficient.

We converted the final reduced data from $T_{A}^{*}$ to $T_{\mathrm{MB}}$ using a main beam efficiency of $\eta_{\mathrm{MB}}=0.61$. The final reduced data were smoothed to $0.85 \mathrm{~km} \mathrm{~s}^{-1}$, resulting in a line sensitivity of $\sim 0.3 \mathrm{~K}$. Since the SCUBA-2 and HARP data were observed at the same facility, both data were taken at very similar resolutions $\left(\sim 14^{\prime \prime}\right)$. Having both the continuum and line data at the same resolution is very advantageous. Sun et al. (2006) made a more complete CO (3-2) survey of the entire Perseus cloud with the KOSMA $3 \mathrm{~m}$ telescope. These data, however, have much lower spatial resolution $\left(\sim 82^{\prime \prime}\right)$, and therefore comparisons to the HARP or SCUBA-2 data at $\sim 14^{\prime \prime}$ are difficult.

\section{RESULTS}

Figure 2 shows the observations at $160 \mu \mathrm{m}, 450 \mu \mathrm{m}, 500 \mu \mathrm{m}$, and $850 \mu \mathrm{m}$ for the central B1 region. Ground-based submillimeter continuum detectors like SCUBA-2 contend with rapidly varying atmospheric signals that greatly affect the detection of radiation. To remove the atmospheric emission, we effectively filter out all large-scale structure from the maps, including the large-scale astronomical signals. For our SCUBA-2 data, this effective spatial filtering corresponds to all angular scales of $\gtrsim 2.5$ based on tests of the mapmaker to recover Gaussians of various sizes (see Appendix A for more details). Therefore, we believe that structures of $\lesssim 2$ '.5 are recovered fully by SCUBA-2. Conversely, space-based instruments like those on board Herschel are free from these limitations and can recover the diffuse largescale emission. For our Herschel observations, we can recover spatial scales within half a scan length $(\sim 1.5)$.

Another key difference between the SCUBA-2 and Herschel observations is resolution. The Herschel resolutions are $\sim 8 . .4,13 . .5,18^{\prime \prime} .2,24.9$, and 36.3 at $70 \mu \mathrm{m}, 160 \mu \mathrm{m}, 250 \mu \mathrm{m}$, $350 \mu \mathrm{m}$, and $500 \mu \mathrm{m}$, respectively, for parallel mode observations at 60 arcsec s ${ }^{-1}$ scan rates. Comparatively, the SCUBA-2 resolutions are $\sim 9^{\prime \prime}$ and $14^{\prime \prime}$ for the $450 \mu \mathrm{m}$ and $850 \mu \mathrm{m}$ bands, respectively. For clustered environments, the gain in resolution brought about by SCUBA-2 at these long wavelengths is important for measuring the properties of compact sources. For instance, some of the compact objects within the central B1 region are blended at the longer Herschel wavelengths but separated out with the SCUBA-2 observations (e.g., see the B1-bN and B1-bS cores in Figure 2).

\subsection{SCUBA-2 and HARP Analyses}

Molecular line contamination is significant if the line flux measured over the continuum band boosts the continuum signal over the expected value from dust emission alone (White et al.
2003). In cold star-forming regions, line contamination is most prominent from rotational transitions of $\mathrm{CO}$, the second most abundant molecule in molecular clouds. While the Herschel data are largely free of line contamination, ${ }^{21}$ the SCUBA-2 $850 \mu \mathrm{m}$ band is susceptible to contamination from the CO (3-2) line transition at $345.796 \mathrm{GHz}$, which lies in the middle of the band and is commonly detected in star-forming regions. Additionally, $\mathrm{CO}$ (6-5) line emission at $691.473 \mathrm{GHz}$ can contaminate the $450 \mu \mathrm{m}$ band; see Appendix B. For a recent summary of the effects of line contamination on observed submillimeter fluxes, see Drabek et al. (2012).

Since the $850 \mu \mathrm{m}$ continuum bandpass is very wide ( $\sim 35 \mathrm{GHz}$ ), molecular lines need to be both bright and broad to make significant contributions to the observed continuum emission (Johnstone et al. 2003). We determined the contribution of $\mathrm{CO}$ (3-2) line emission to the continuum using the same technique as Drabek et al. (2012). For more details, see Appendix B. Unlike SCUBA-2, the HARP CO (3-2) line data recover largescale structures since they were obtained using a reference position free of line emission at a much larger angular distance from the target positions. Therefore, we ran the integrated intensity $\mathrm{CO}$ (3-2) map through the SCUBA-2 reduction pipeline to produce a filtered $\mathrm{CO}$ flux map that includes only emission on the same spatial scales as the $850 \mu \mathrm{m}$ data (see Appendix A). The filtered CO (3-2) line emission contributed $\lesssim 70 \mathrm{mJy}^{\text {beam }}{ }^{-1}$ $\left(\lesssim 100 \mathrm{~K} \mathrm{~km} \mathrm{~s}^{-1}\right)$ to the $850 \mu \mathrm{m}$ continuum emission with the most significant contributions toward the outflows associated with the protostellar cores B1-c and B1-d.

Figure 3 compares the $850 \mu \mathrm{m}$ map before and after the $\mathrm{CO}$ (3-2) line contamination was removed. In the left panel, the two lobes of $\mathrm{CO}(3-2)$ emission along the northern outflow clearly dominate the continuum flux, contributing up to $\sim 90 \%$ of the total $850 \mu \mathrm{m}$ flux at the positions of the outflow. Aside from these outflows, the corrected $850 \mu \mathrm{m}$ map (right panel) shows little difference with the original map (left). The CO (3-2) line emission contributes $\lesssim 15 \%$ of the total $850 \mu \mathrm{m}$ flux for the central region of $\mathrm{B} 1$ and $\lesssim 1 \%$ for the rest of the $\mathrm{B} 1$ clump.

Hereafter, the observed $850 \mu \mathrm{m}$ data have been corrected for CO (3-2) line contamination. For comparisons with Herschel data, we convolved both the CO-corrected $850 \mu \mathrm{m}$ map and the observed $450 \mu \mathrm{m}$ map to the $500 \mu \mathrm{m}$ resolution of 36.3 . For the $450 \mu \mathrm{m}$ map, both the main beam and error beam were considered in the convolution, ${ }^{22}$ whereas for the $850 \mu \mathrm{m}$ map, we found that the relative power in the error beam was not as significant.

\subsection{Herschel-only Results}

We used the PACS $160 \mu \mathrm{m}$ and the SPIRE $250 \mu \mathrm{m}, 350 \mu \mathrm{m}$, and $500 \mu \mathrm{m}$ data to estimate the dust properties in B1. Since the PACS $70 \mu \mathrm{m}$ band can trace non-equilibrium emission from very small dust grains (Martin et al. 2012) and warm dust emission from protostellar sources (as opposed to the cool envelope traced by the longer wavelengths), we do not include those data in our analysis. First, we corrected the arbitrary zeropoint flux offset in each band using Planck HFI and IRAS data

\footnotetext{
21 The $350 \mu \mathrm{m}$ band can be contaminated by the high excitation transition $\mathrm{CO}$ (7-6) at $806.652 \mathrm{GHz}$, though we believe such contamination should be negligible toward B1. See Appendix B.

22 For the $450 \mu \mathrm{m}$ map, the error beam has an FWHM of $\sim 25^{\prime \prime}$, which is smaller than the $500 \mu \mathrm{m}$ beam. We convolved a two-component Gaussian beam with a main component of 7..9 and an error component of $25^{\prime \prime}$ to $34^{\prime \prime} .25$ for an effective resolution of 36 ". 3 . Without considering the error beam, the $450 \mu \mathrm{m}$ fluxes would be overestimated by $\lesssim 10 \%$.
} 

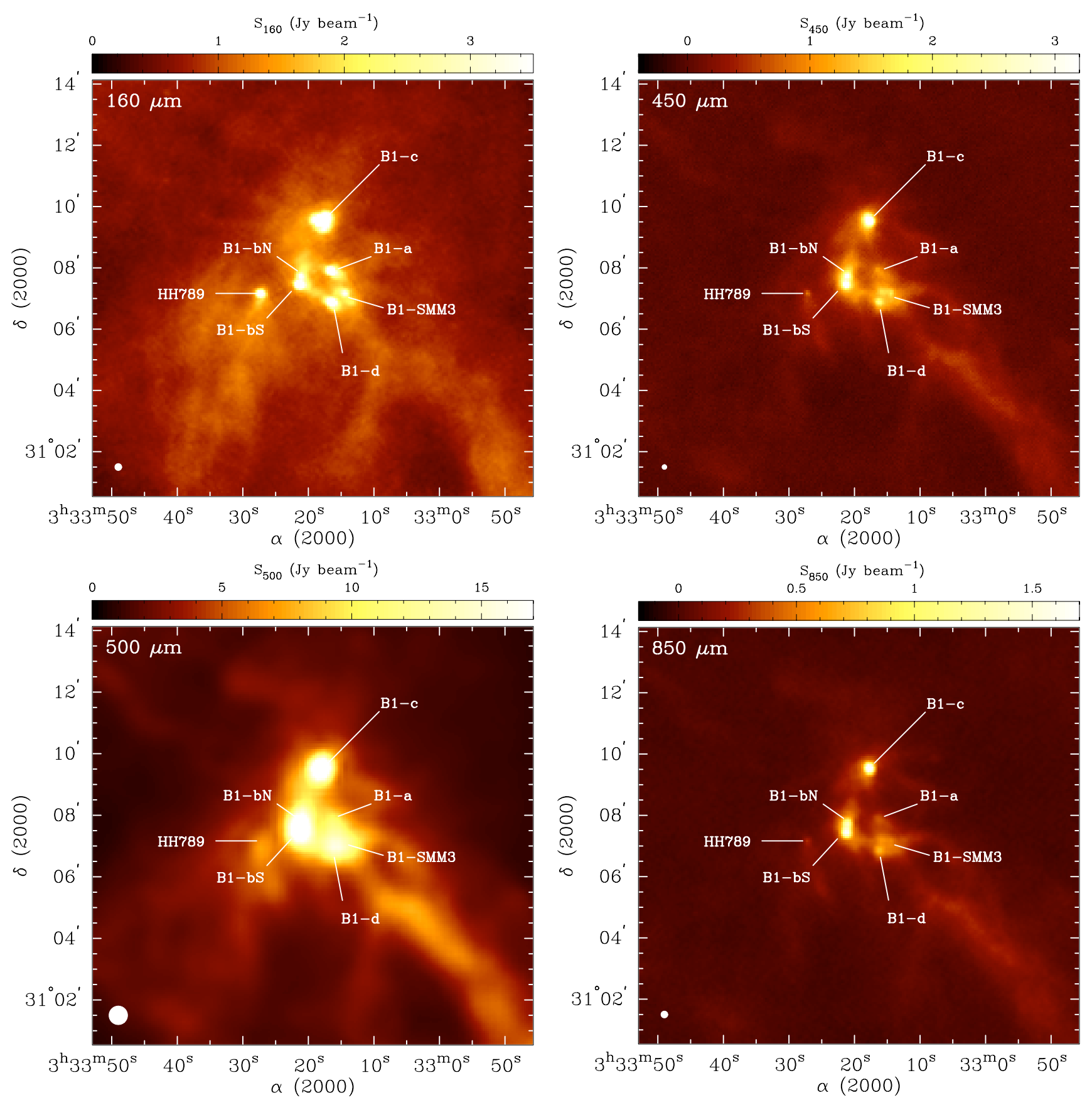

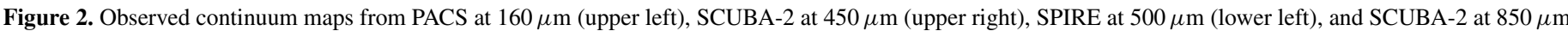

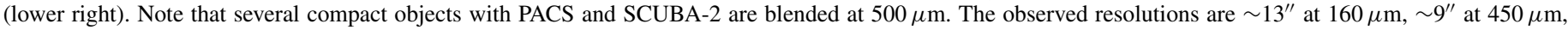
$\sim 36^{\prime \prime}$ at $500 \mu \mathrm{m}$, and $\sim 14^{\prime \prime}$ at $850 \mu \mathrm{m}$. These maps reveal several known sources in the central B1 region (see Jørgensen et al. 2007; Hatchell et al. 2007).

following the method proposed in Bernard et al. (2010). Second, we convolved each map to the same resolution $\left(36^{\prime \prime} .3\right.$, or the $500 \mu \mathrm{m}$ beam FWHM) and projected all maps onto a common grid of $14^{\prime \prime}$ pixels.

For the PACS and SPIRE bands, minor color correction factors $(\lesssim 3 \%)$ are necessary (for more details see Pezzuto et al. 2012 and Appendix C). Table 1 lists our adopted color correction factors and uncertainties, assuming $\beta \approx 1.5-2.5$ and $T \approx 10 \mathrm{~K}-15 \mathrm{~K}$. Additionally, we estimated the pixel-topixel rms sensitivity by selecting several areas relatively free of diffuse emission in the Perseus West map (see Figure 1 in Sadavoy et al. 2012). Table 1 lists the approximate $1 \sigma$ rms uncertainties at the native resolution of each band and for the convolved maps ( $36^{\prime \prime} .3$ resolution). Since the selected regions were not entirely free of emission, these sensitivity measurements can be considered upper limits to the true map noise. These map sensitivities also depend greatly on the processing, such as the mapmaker. The observed $1 \sigma$ rms noise estimates are within a factor of $\sim 2$ of the expected $1 \sigma \mathrm{rms}$ noise errors according to the Herschel observation planning tool (HSpot). ${ }^{23}$

Table 1 excludes additional errors from flux calibration. Flux calibration depends on a number of factors, including the calibration files used in the reduction and the mapmaker.

\footnotetext{
23 HSpot is a joint development by the Herschel Science Ground Segment Consortium, consisting of ESA, the NASA Herschel Science Center, and the HIFI, PACS, and SPIRE consortia.
} 

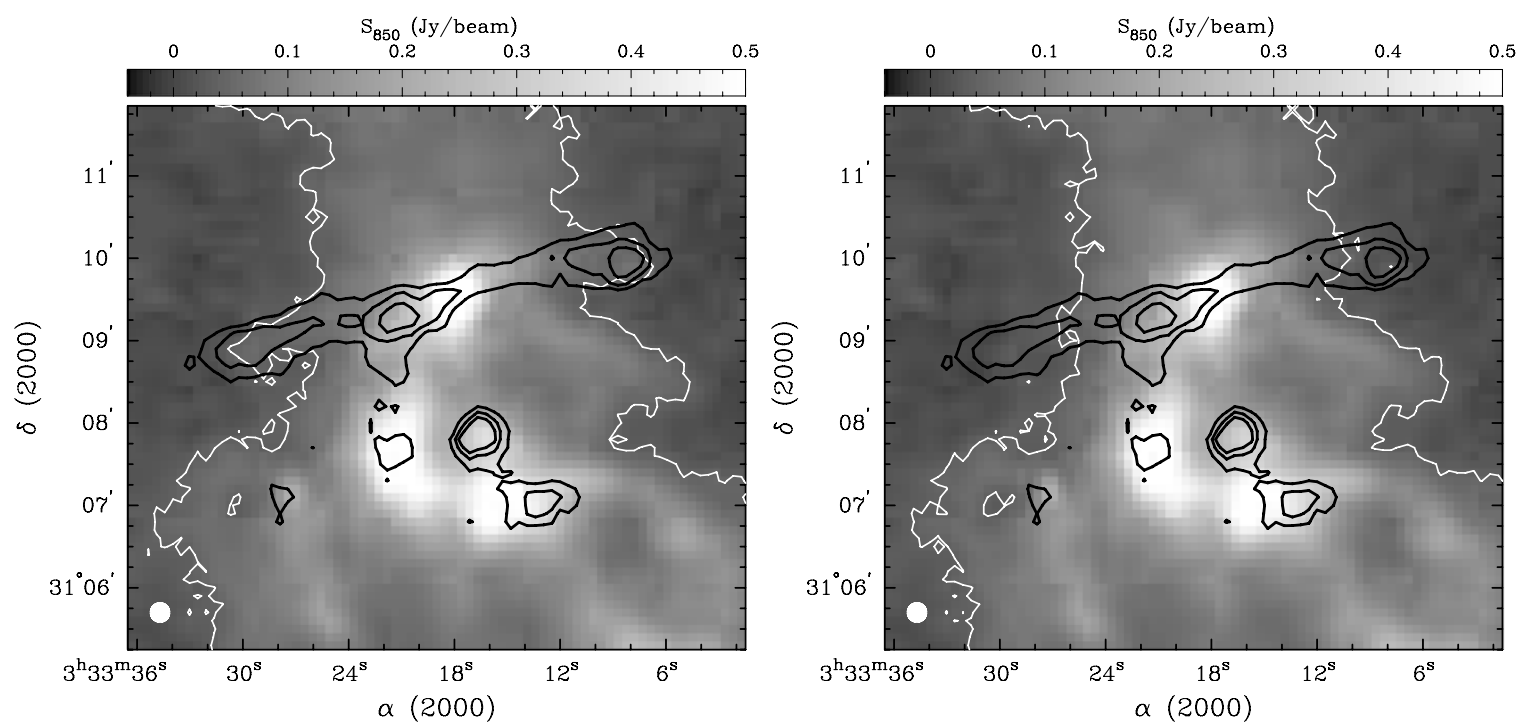

Figure 3. Comparisons between $850 \mu \mathrm{m}$ continuum emission and CO (3-2) line emission. Left: background image shows the uncorrected $850 \mu \mathrm{m}$ continuum map. The black contours correspond to CO (3-2) line emission at levels of $10 \mathrm{mJy}$ beam ${ }^{-1}, 20 \mathrm{mJy} \mathrm{beam}^{-1}$, and $30 \mathrm{mJy} \mathrm{beam}^{-1}$. The CO data were filtered using the SCUBA-2 pipeline (see Appendix A). The white contours show $850 \mu \mathrm{m}$ continuum emission at $30 \mathrm{mJy} \mathrm{beam}^{-1}$. Right: background image shows the $850 \mu \mathrm{m}$ continuum map with the filtered CO line emission subtracted out. The white contours show corrected $850 \mu \mathrm{m}$ emission at $30 \mathrm{mJy} \mathrm{beam}^{-1}$. The SCUBA-2 and HARP data have a common resolution of $\sim 14{ }^{\prime \prime} 2$, shown as a white circle.

Table 1

Adopted Color Corrections and Flux Uncertainties

\begin{tabular}{lcccc}
\hline \hline Band & $160 \mu \mathrm{m}$ & $250 \mu \mathrm{m}$ & $350 \mu \mathrm{m}$ & $500 \mu \mathrm{m}$ \\
\hline Color correction $^{\mathrm{a}}$ & 1.01 & 1.02 & 1.01 & 1.03 \\
Color uncertainty $^{\mathrm{b}}$ & $5 \%$ & $0.8 \%$ & $1 \%$ & $2 \%$ \\
$\mathrm{rms}_{\nu}\left(\mathrm{mJy} \mathrm{beam}^{-1}\right)^{\mathrm{c}}$ & 20 & 18 & 15 & 20 \\
$\mathrm{rms}_{36.3}\left(\mathrm{mJy} \mathrm{beam}^{-1}\right)^{\mathrm{d}}$ & 80 & 60 & 30 & 20 \\
\hline
\end{tabular}

Notes.

a Average color corrections for each band, where $S_{\text {corrected }}=\mathrm{CC} \times S_{\text {obs }}$. These values assume $T \approx 10-15 \mathrm{~K}$ and $\beta \approx 1.5-2.5$. See Appendix $\mathrm{C}$.

${ }^{\mathrm{b}}$ Uncertainties refer to the fractional uncertainty in the average color correction based on the range of accepted color corrections. These uncertainties do not include the calibration uncertainties.

${ }^{\mathrm{c}}$ Approximate $1 \sigma \mathrm{rms}$ noise in the Herschel maps for relatively blank regions at the native resolution of each band.

${ }^{\mathrm{d}}$ Approximate $1 \sigma \mathrm{rms}$ noise in the convolved Herschel maps for relatively blank regions at 36.3 resolution.

Based on point sources, the flux calibrations uncertainties are roughly 5\% for PACS ${ }^{24}$ and $7 \%$ for SPIRE. ${ }^{25}$ Since we are interested in extended emission and our observations were taken with the fast $\left(60 \operatorname{arcsec} \mathrm{s}^{-1}\right)$ scan rate resulting in elongated beams, we adopted $10 \%$ flux calibration errors for both PACS and SPIRE. To simulate these uncertainties, we generated 1000 random correction factors following a Gaussian with a mean of 1.0 and a half-width at half-maximum of 0.1 . Since the flux calibrations are correlated for each instrument (M. Griffin 2012, private communication), we assumed the same flux calibration corrections for the three SPIRE bands and a separate randomly generated set of corrections for the PACS $160 \mu \mathrm{m}$ band. We applied the randomly selected PACS and SPIRE calibration correction factors appropriately across the maps. For each pair

\footnotetext{
${ }^{24}$ PACS flux calibration can be found in the "Assessment analysis of the extended emission calibration for the PACS red channel,"

http://herschel.esac.esa.int/twiki/bin/view/Public/PacsCalibrationWeb. 25 SPIRE flux calibration can be found in the SPIRE Observers' Manual (2011), HERSCHEL-DOC-0798, ver. 2.4, http://herschel.esac.esa.int/Docs/SPIRE/pdf/spire_om.pdf.
}

of correction factors, we fit the resulting SEDs pixel by pixel to identify the broad distribution of best-fit SED parameters within the calibration uncertainties.

We fitted the SEDs of individual pixels using the IDL program mpfitfun, which performs an iterative least-squares comparison between a set of data and a model function until a best fit is achieved (Markwardt 2009). We fit each SED with the modified blackbody function,

$$
I_{v}=\kappa_{\nu_{0}}\left(v / \nu_{0}\right)^{\beta} B_{v}(T) \Sigma,
$$

where $\kappa_{\nu_{0}}$ is a reference dust opacity per unit gas and dust mass at reference frequency $\nu_{0}, \beta$ is the dust emissivity power-law index, $B_{v}$ is the blackbody function, $T$ is the dust temperature, and $\Sigma=\mu m_{H} N\left(\mathrm{H}_{2}\right)$ is the gas mass column density of material with mean molecular mass $\mu m_{H}$ and gas column density $N\left(\mathrm{H}_{2}\right)$. For consistency with other papers from the Herschel GBS (e.g., André et al. 2010), we assumed that $\kappa_{\nu_{0}}=0.1 \mathrm{~cm}^{2} \mathrm{~g}^{-1}$ at $v_{0}=1000 \mathrm{GHz}$ (Hildebrand 1983). We adopt a mean molecular weight per unit hydrogen mass of $\mu=2.8$ for a cloud of $71 \%$ molecular hydrogen gas, $27 \%$ helium, and $2 \%$ metals (e.g., Kauffmann et al. 2008).

Most Herschel GBS analyses (e.g., André et al. 2010; Arzoumanian et al. 2011; Sadavoy et al. 2012) fit SEDs across the $160-500 \mu \mathrm{m}$ Herschel bands assuming $\beta=2$ (Hildebrand 1983). To test this assumption, we used Equation (1) to fit the Herschel $160-500 \mu \mathrm{m}$ data assuming (1) $\beta=1.5$, (2) $\beta=2.0$, (3) $\beta=2.5$, and (4) $\beta$ is a free variable. We applied the color correction factors (see Table 1) and the random distribution of calibration correction factors to the observed emission. For the flux uncertainties, we added in quadrature the color calibration uncertainties and the map sensitivities (see Table 1). Figure 4 shows the distributions of SED-derived parameters for a single example pixel toward the B1-a core. For our sample pixel, when $\beta$ is fixed, the temperature distributions are narrow despite the wide range in calibration correction factors. The mean temperature, however, varies from $T \approx 14 \mathrm{~K}$ for $\beta=1.5$ to $T \approx 10.5 \mathrm{~K}$ for $\beta=2.5$. Allowing $\beta$ to vary produces broad Gaussian-like temperature and $\beta$ distributions with mean values 

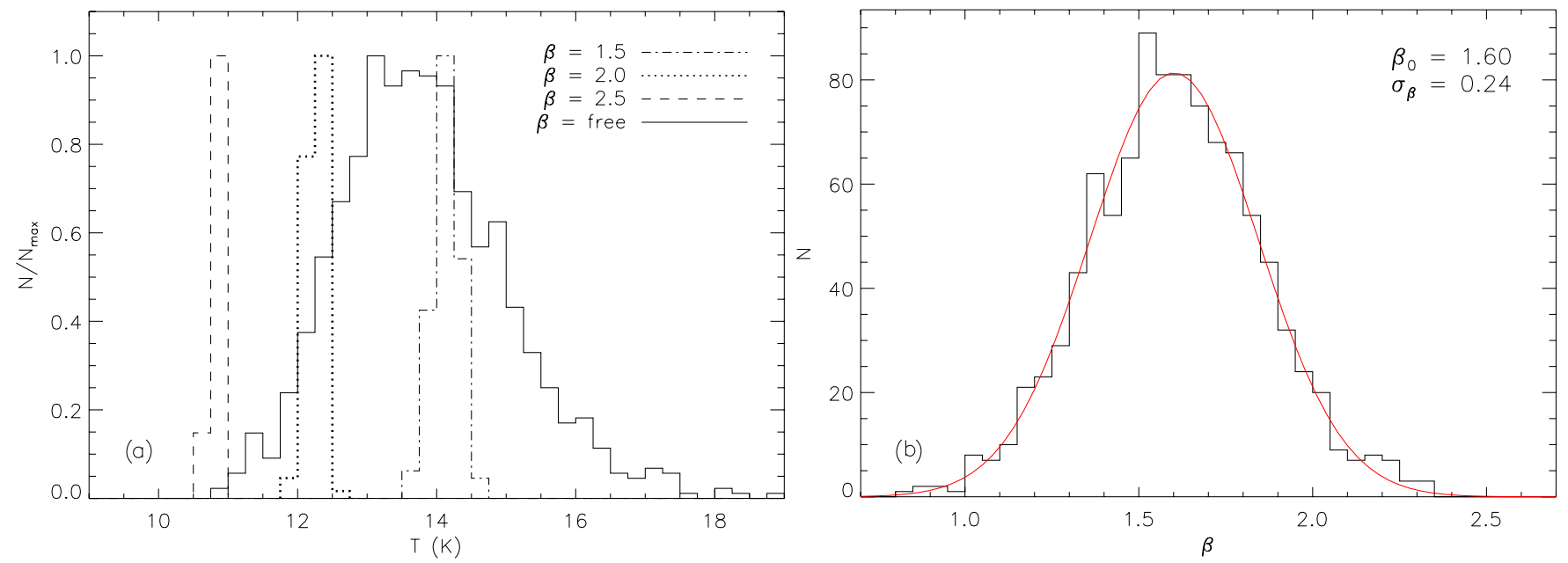

Figure 4. Sample results from SED fitting toward a pixel in the B1-a core. We fit the observed SEDs after applying our color correction factors and random calibration correction factors (see the text). For each calibration correction factor, we fit the modified dust emission with a dusty blackbody model assuming $\beta=1.5,2.0,2.5$, and free $\beta$. We show the (a) relative temperature distributions for each of these cases of $\beta$ and (b) the $\beta$ distribution when unrestricted. We fit the $\beta$ distribution with a Gaussian (gray curve; red curve online).

(A color version of this figure is available in the online journal.)

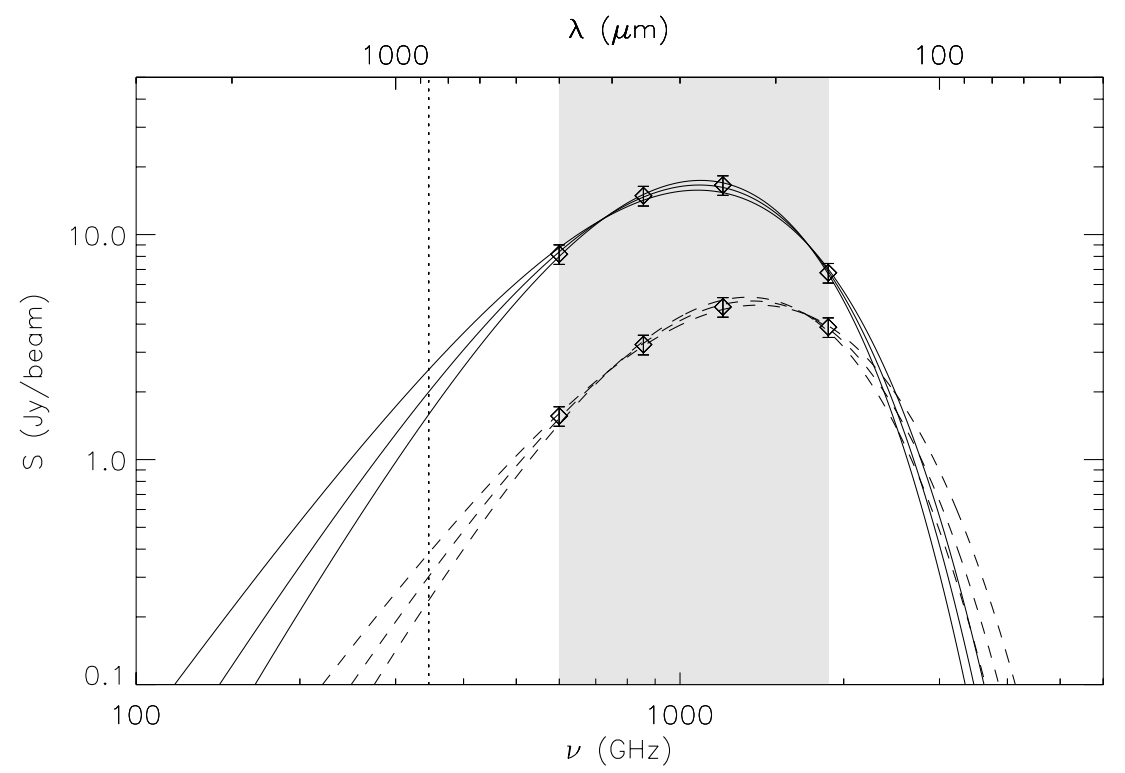

Figure 5. Sample modified blackbody profiles for dust emission corresponding to (solid) $T=10 \mathrm{~K}, N\left(\mathrm{H}_{2}\right)=10^{23} \mathrm{~cm}^{-2}$, and $\beta=2.25$; and (dashed) $T=14 \mathrm{~K}$, $N\left(\mathrm{H}_{2}\right)=6 \times 10^{21} \mathrm{~cm}^{-2}$, and $\beta=1.75$. For both cases, we fit the generated SEDs (diamonds) with (from left to right) $\beta=1.5,2.0$, or 2.5 . The Herschel $160-500 \mu \mathrm{m}$ bands cover the region highlighted by the solid gray box. The dotted line corresponds to $850 \mu \mathrm{m}$. At short wavelengths (i.e., $\lambda \lesssim 100 \mu \mathrm{m}$ ), the dust emission may become optically thick, and thus, the SED curves could be independent of $\beta$ at these wavelengths. For simplicity, we do not consider optically thick dust emission at short wavelengths. Thus, $\beta$ cannot be constrained with short-wavelength observations and instead, long-wavelength observations are necessary.

of $13.7 \mathrm{~K} \pm 1.1 \mathrm{~K}$ and $1.60 \pm 0.24$, respectively. These broad distributions suggest that the Herschel calibration uncertainties result in significant uncertainties for the SED parameters.

Furthermore, Figure 5 highlights the $\beta$-temperature degeneracy of SED fitting with the Herschel bands only. First, we generated fluxes assuming (1) $T=10 \mathrm{~K}, N\left(\mathrm{H}_{2}\right)=10^{23} \mathrm{~cm}^{-2}$, and $\beta=2.25$; and (2) $T=14 \mathrm{~K}, N\left(\mathrm{H}_{2}\right)=6 \times 10^{21} \mathrm{~cm}^{-2}$, and $\beta=1.75$. Second, we fitted the generated Herschel band fluxes with our dusty blackbody models assuming $\beta=1.5,2.0$, and 2.5 and no calibration uncertainties. The solid curves show the best-fit SED models to the dust emission for the first case and the dashed curves show the best-fit SED models for the second case. For both cases, the generated data were moderately well fit by all three values of $\beta$. Note that the Herschel bands cover the region illustrated by the gray box where the model curves are largely degenerate. The error bars indicate an uncertainty of $10 \%$ to illustrate the range of uncertainty from the calibration. (Recall that instrument calibration is correlated such that the same calibration correction will be required for the three SPIRE bands.) Conversely, at longer wavelengths, the SED models are more distinct, and thus the SED parameters are much better constrained. Since we have longer wavelength data (i.e., $850 \mu \mathrm{m}$ ), we may circumvent the degeneracy between $\beta$ and temperature (see Section 4).

\section{DETERMINING $\beta$}

As discussed in Section 3.1, the SCUBA-2 observations do not recover large-scale emission, and that emission can be significant. For example, the residuals between a prediction 

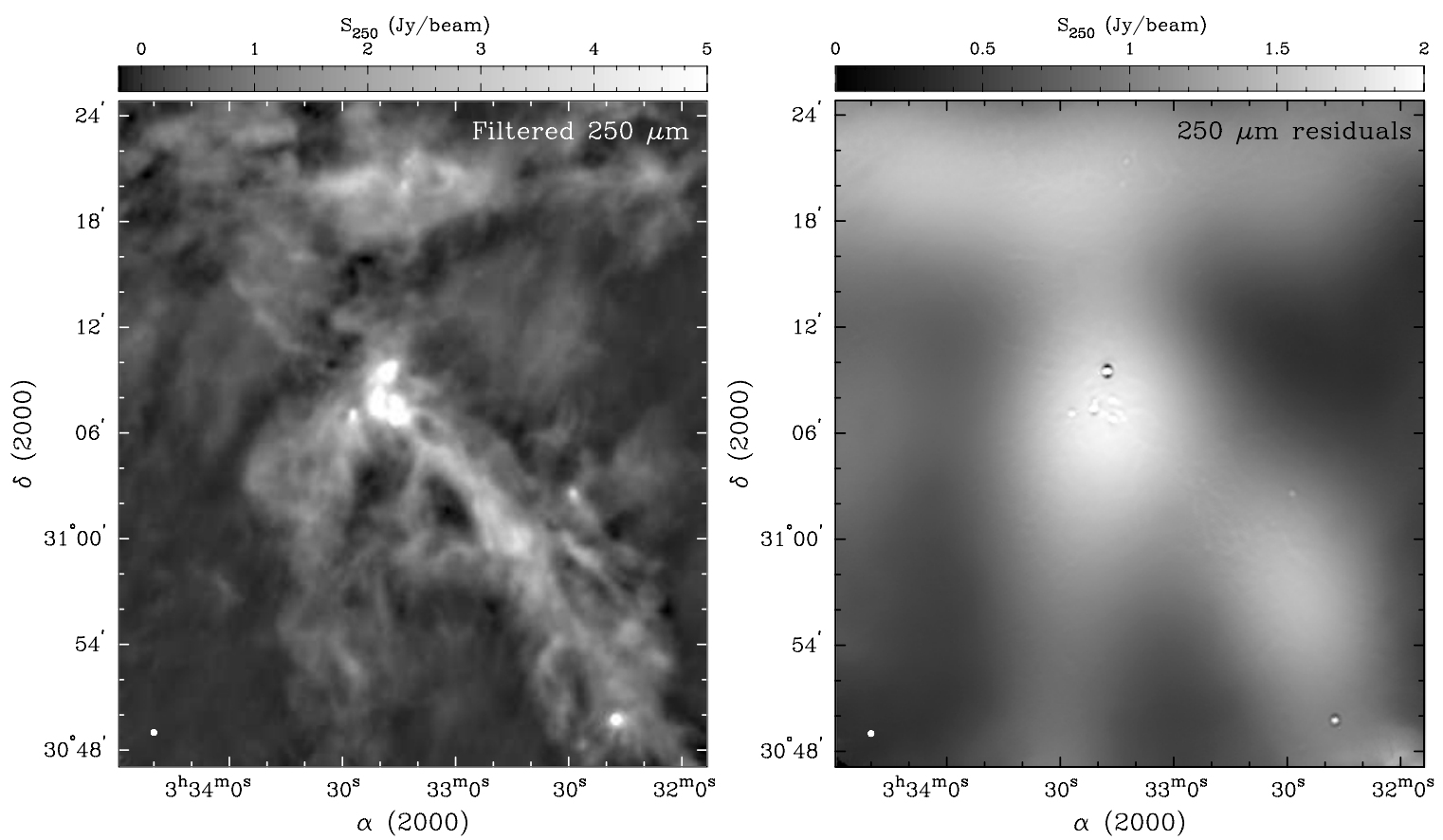

Figure 6. Comparison between the filtered Herschel map at $250 \mu \mathrm{m}$ and the filtered-out large-scale emission (using the SCUBA-2 mapmaker; see Appendix A). Map resolutions are $18^{\prime \prime}$.

of unfiltered $850 \mu \mathrm{m}$ emission (i.e., from SED fitting of the Herschel data assuming $\beta=2$ ) and the observed $850 \mu \mathrm{m}$ dust emission suggest that the observed fluxes at $850 \mu \mathrm{m}$ could be missing $\gtrsim 30 \%$ of the total emission. Therefore, direct comparisons between PACS+SPIRE and SCUBA-2 observations can be misleading.

In this section, we explore four different techniques to determine $\beta$ toward B1 using the SCUBA-2 data. In Section 4.1, we determined $\beta$ using filtered dust emission maps, and in Section 4.2 , we determined $\beta$ using unfiltered maps. Since filtered maps do not include any large-scale structure, there is no reason to expect a priori that $\beta$ will be the same for filtered and unfiltered maps. For example, the extended emission may trace more of the warmer, diffuse cloud structure than the cold, dense structures associated with cores, therefore broadening the SED and lowering $\beta$ (Martin et al. 2012). If true, then the absence of the large-scale structure may result in more pronounced variations in $\beta$ toward the small-scale features in the filtered maps than in the unfiltered maps. For all fits to the SEDs, we used the following procedure unless stated otherwise.

1. We applied the color correction factors in Table 1 to the Herschel bands.

2. We adopted flux errors as the quadrature sum of the $1 \sigma$ rms errors and the uncertainty of the color corrections (see Table 1).

3. We generated different sets of 1000 random calibration correction factors following a Gaussian distribution to represent a $10 \%$ or $50 \%$ calibration uncertainty. We generated a separate set of calibration correction factors for PACS, SPIRE, SCUBA-2 at $450 \mu \mathrm{m}$, and SCUBA- 2 at $850 \mu \mathrm{m}$.

4. We fit SEDs following a minimization of $\chi^{2}$ routine. We used the modified blackbody model in Equation (1) and the IDL mpfitfun routine to minimize the $\chi^{2}$ fit.

5. For the filtered maps (see Section 2.2), we only considered regions with $\geqslant 10 \sigma$ intensities at $160-850 \mu \mathrm{m}$ at $36^{\prime \prime}$ resolution (excluding the $450 \mu \mathrm{m}$ band). The filtered maps are only well measured within the masked regions used in the reduction (see Section 2.2). Since the $450 \mu \mathrm{m}$ data have a higher $1 \sigma \mathrm{rms}$ noise level $\left(\sim 100 \mathrm{mJy}\right.$ beam $\left.^{-1}\right)$, these regions include $450 \mu \mathrm{m}$ emission that is $<5 \sigma$.

\section{1. $\beta$ Using Filtered Maps}

For our first case (hereafter the Filtered Case), we removed the large-scale emission from the Herschel observations by applying the SCUBA-2 reduction pipeline to the observed Herschel maps as we did with the $\mathrm{CO}(3-2)$ line data (see Appendix A for more information). This technique has the advantage of forcing all the data to include the same range of spatial scales, and thus $\beta$ and temperature can be determined from directly fitting the SEDs of the $160-850 \mu \mathrm{m}$ emission. Unlike with our SCUBA-2 data, we can compare the filtered and unfiltered versions of the Herschel maps and see how the SCUBA-2 mapmaker removes largescale emission. Figure 6 compares the filtered SPIRE $250 \mu \mathrm{m}$ map with the filtered-out large-scale emission. Note that a few artifacts remain at the locations of the brightest cores. These artifacts are very localized, and thus become negligible $(<5 \%$ of the observed emission) when the filtered maps are convolved to 36.3 resolution. As this paper is being written, the SCUBA-2 reduction pipeline is still evolving. Thus, while some artifacts may be introduced in this version of the SCUBA-2 reduction pipeline, future versions of the pipeline should improve the filtering.

For our second case (hereafter the SCUBA-2 Ratio Case), we determined $\beta$ using the $450 \mu \mathrm{m}$ and $850 \mu \mathrm{m}$ bands only, i.e., without Herschel data (e.g., see Hill et al. 2006; Hatchell et al. 2013). Using Equation (1), the ratio of the fluxes at $450 \mu \mathrm{m}$ and $850 \mu \mathrm{m}$ is

$$
\frac{I_{450}}{I_{850}}=\left(\frac{v_{450}}{v_{850}}\right)^{(\beta+3)}\left[\frac{\exp \left(h v_{850} / k T\right)-1.0}{\exp \left(h v_{450} / k T\right)-1.0}\right] .
$$

Therefore, $\beta$ can be determined from the ratio of $450 \mu \mathrm{m}$ to $850 \mu \mathrm{m}$ emission for a given temperature. For simplicity, we 


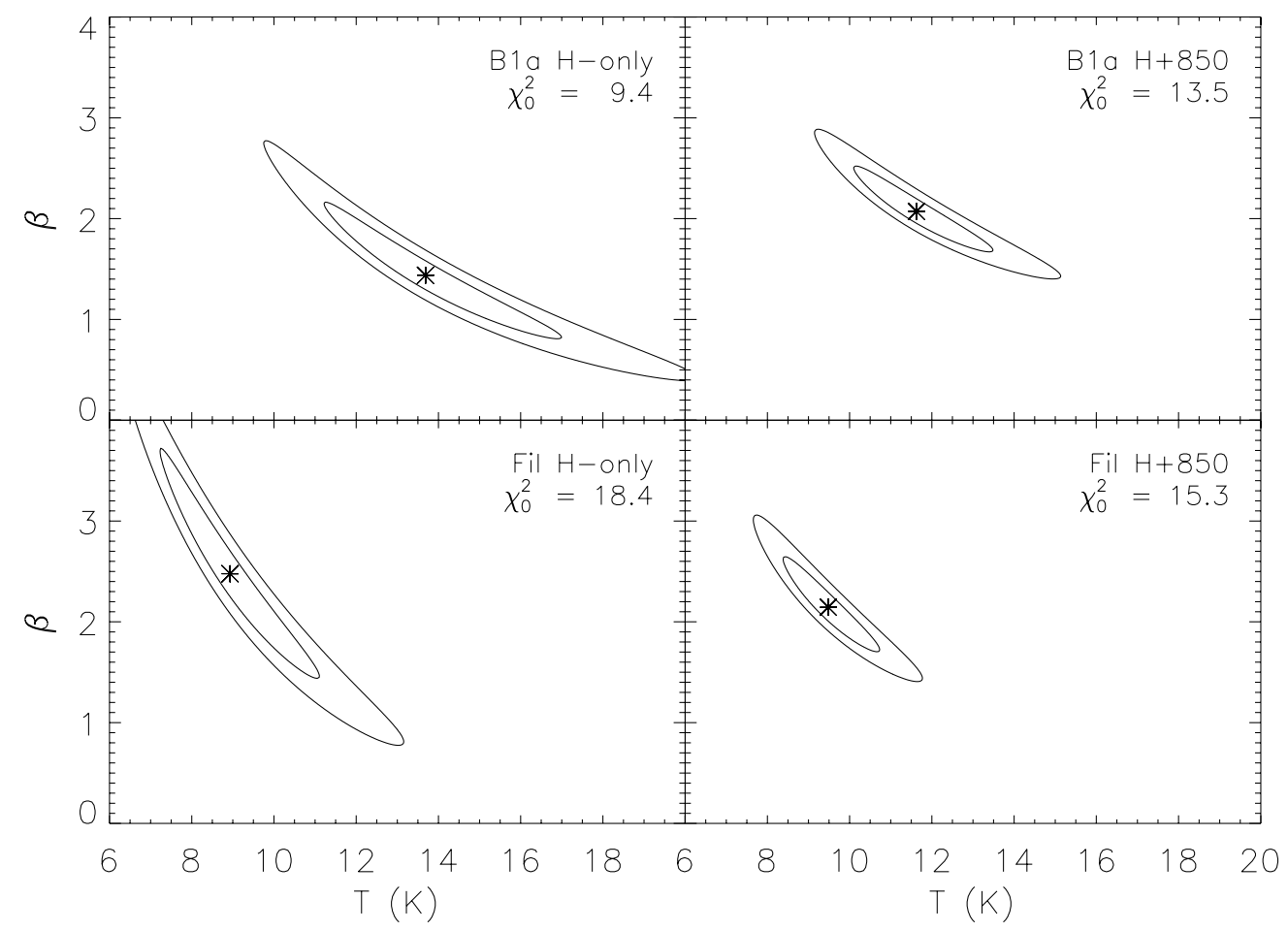

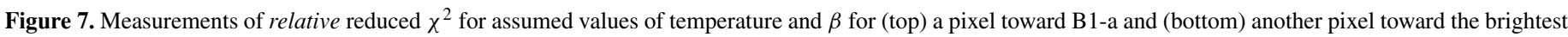

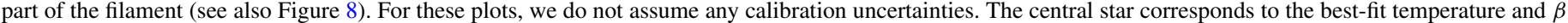

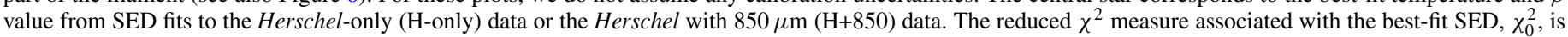

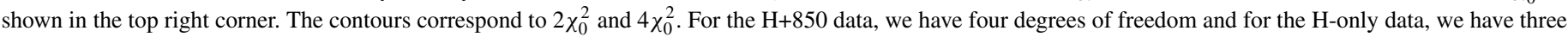
degrees of freedom.

find $\beta$ for assumed line-of-sight temperatures. We do not naively expect the line-of-sight temperature to be constant, particularly since the B1 cluster contains known protostellar sources (i.e., B1-c, see Figure 3). Additionally, we also assume that the $450 \mu \mathrm{m}$ and $850 \mu \mathrm{m}$ maps include the same range of spatial scales.

For the Filtered Case, Figure 7 shows contour plots of reduced $\chi^{2}$ assuming temperatures of $5 \mathrm{~K} \leqslant T \leqslant 20 \mathrm{~K}$ and $\beta$ values of $0.5 \leqslant \beta \leqslant 4.0$. These plots highlight the uncertainties from the minimization of $\chi^{2}$ technique only. For clarity, we do not include the calibration uncertainties for these plots. (We note, however, that the calibration uncertainties will only increase the accepted range of $\beta$ and temperature values and would not change the behavior of the SED fitting itself.) For simplicity, we show the results for a pixel toward B1-a and a pixel toward the brightest part of the filament, though these sample pixels represent the typical behavior for B1-a and the filament. We compare the results from SED fitting to the Herschel bands alone (H-only) and the Herschel bands with the $850 \mu \mathrm{m}$ data $(\mathrm{H}+850)$, where the contours correspond to $2 \chi_{0}^{2}$ and $4 \chi_{0}^{2}$, and $\chi_{0}^{2}$ is the reduced $\chi^{2}$ measure from the best-fit SED to the corresponding data. Thus, Figure 7 represents the relative uncertainties in $\beta$ and temperature from SED fits using the minimization of $\chi^{2}$ technique. By adding the $850 \mu \mathrm{m}$ flux as a new constraint, we see significant improvements in our ability to determine $\beta$ and temperature. For example, we find improvements in the uncertainty of $\beta$ by a factor of $\sim 2$, and improvements in the uncertainty of temperature by $\sim 40 \%$ with the $850 \mu \mathrm{m}$ data.

In addition to temperature and $\beta$ uncertainties from the fitting technique, each instrument has a $10 \%$ flux calibration error. As in Section 3.2, we applied calibration correction factors within a Gaussian distribution to represent the flux calibration errors for each instrument. Figure 8 shows the mean $\beta$ values and two example SEDs for the H-only and $\mathrm{H}+850$ cases. For the $\mathrm{H}$-only case, we found more extreme values of $\beta$ toward the dense cores and filament. For example, the $\mathrm{H}$-only fits give $\beta \approx$ 1.5 toward $\mathrm{B} 1-\mathrm{a}$ and $\beta \approx 2.5$ toward the filament, whereas the $\mathrm{H}+850$ fits give $\beta \approx 2.0$ toward both. Similarly, the H-only fits overestimated the temperature by $\sim 2 \mathrm{~K}$ toward B1-a and underestimated the temperature by $\lesssim 1 \mathrm{~K}$ toward the filament compared to the $\mathrm{H}+850$ results. In the bottom panels of Figure 8 , we show the SEDs for the sample pixels toward B1-a and the southwest filament, corresponding to the same example pixels in Figure 7. With the $850 \mu \mathrm{m}$ band included, we found a significant difference in the absolute value of $\beta$ (see also Figure 7), though the uncertainties related to the calibration correction factors are nearly identical.

Figure 9 shows the distributions of temperature and $\beta$ for the Filtered Case. These plots highlight the change in the absolute value of $\beta$ between the $\mathrm{H}+850$ and $\mathrm{H}$-only fits. For the mean $\beta$ distribution (Figure 9(b)), the H-only $\beta$ distribution peaks at $\beta \approx 2.5$ and the $\mathrm{H}+850$ distribution peaks at $\beta \approx 2.0$. For both the H-only and $\mathrm{H}+850$ fits, the $1 \sigma$ standard deviation errors based on the calibration uncertainties alone are $\sigma_{T} \lesssim 1.5 \mathrm{~K}$ for temperature and $\sigma_{\beta}<0.3$ for $\beta$. Note that these errors are typically less than the errors from the $\chi^{2}$ fitting (Figure 7), suggesting that the constraints on the SED fitting are more significant than uncertainties in the flux calibration. For larger calibration uncertainties at $850 \mu \mathrm{m}$, however, the uncertainties in temperature and $\beta$ due to the calibration become more significant. For example, if we were to assume $50 \%$ calibration errors at $850 \mu \mathrm{m}$, then the $1 \sigma$ standard deviation errors would become $\sigma_{T} \lesssim 3.7 \mathrm{~K}$ and $\sigma_{\beta} \lesssim 0.8$, and thus we cannot well constrain either parameter. Therefore, long-wavelength 


\section{$\beta$}
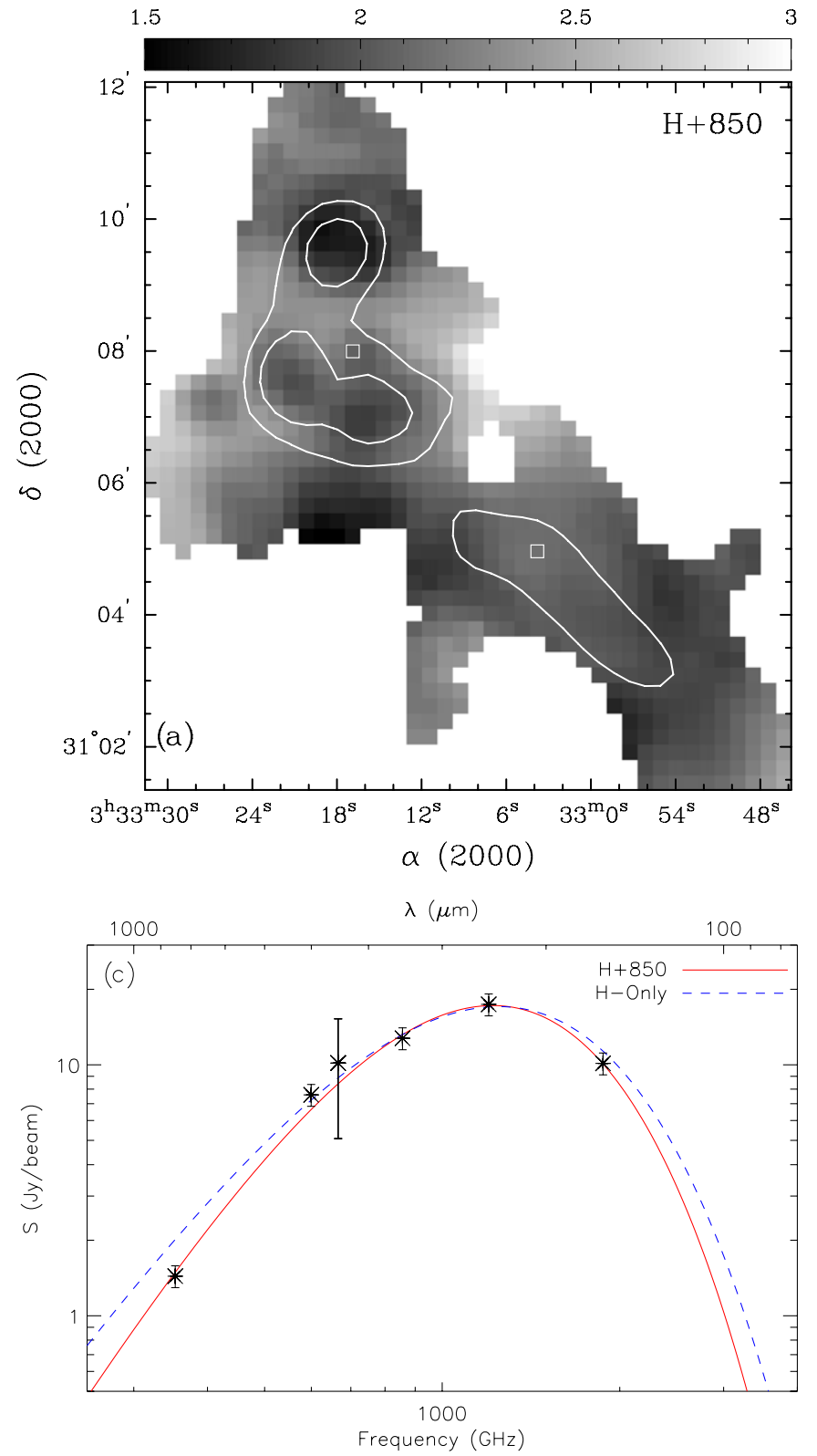
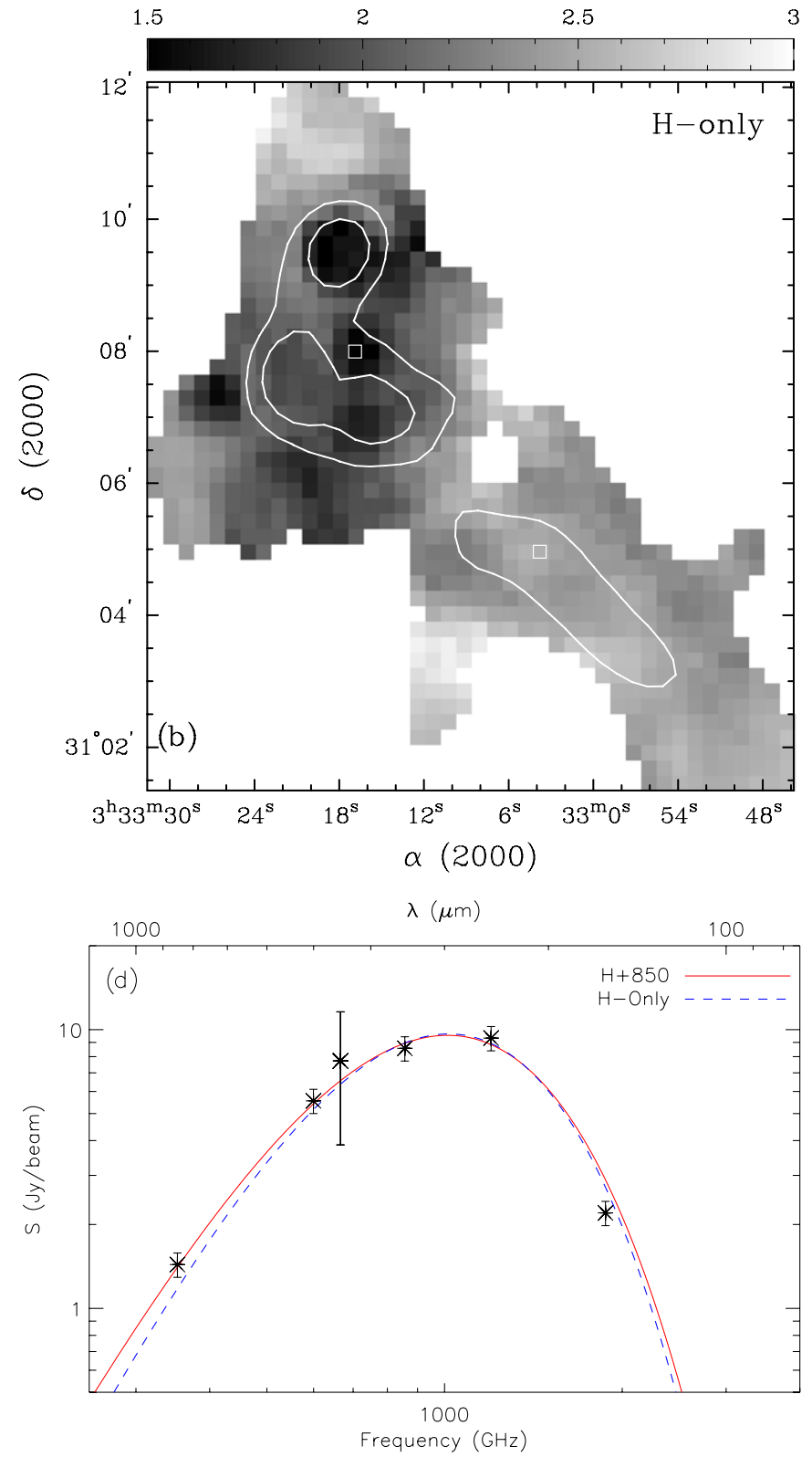

Figure 8. Top: dust emissivity maps toward the B1 main cluster for the Filtered Case. The white contours show $850 \mu \mathrm{m}_{\text {flux levels of } 1 \mathrm{Jy} \text { beam }}{ }^{-1}$ and $2 \mathrm{Jy}_{\text {beam }}{ }^{-1}$ at $36^{\prime \prime}$ resolution. Bottom: sample SEDs from (c) B1a and (d) the southwest filament, each marked by squares in the top panel. The curves show the best-fit SEDs. For the solid curves, we used the $\mathrm{H}+850$ bands, whereas for the dashed curves, we used the $\mathrm{H}$-only bands. The error bars on the data points illustrate the range in flux from the calibration uncertainties. The $450 \mu \mathrm{m}$ band is included for comparison; it was not used in the fitting.

(A color version of this figure is available in the online journal.)

observations can improve the SED fitting only if the calibration uncertainties are relatively low, as achieved with SCUBA-2 at $850 \mu \mathrm{m}$.

Alternatively, with the SCUBA-2 Ratio Case, we can determine $\beta$ if the temperature is known (see Equation (2)). For simplicity, we assumed fixed temperatures across the entire map and for comparison with the Filtered Case, we used the 36.3 convolved maps at $450 \mu \mathrm{m}$ and $850 \mu \mathrm{m}$. Additionally, we generated 1000 random calibration correction factors within a Gaussian distribution assuming a calibration uncertainty of 50\% at $450 \mu \mathrm{m}$ and $10 \%$ at $850 \mu \mathrm{m}$. Figure 10 compares the resulting mean $\beta$ distributions from the SCUBA-2 Ratio Case for the assumed temperatures of $T=9 \mathrm{~K}, 11 \mathrm{~K}$, and $13 \mathrm{~K}$. While the $\beta$ distributions appear to have very similar structures, the distribu- tions themselves are clearly shifted with respect to one another. Using a Kolmogorov-Smirnov test, the $\beta$ distributions at $T=$ $9 \mathrm{~K}$ and $T=11 \mathrm{~K}$ agree within $90 \%$ for a shift of $\Delta \beta \approx 0.4$, and the distributions at $T=11 \mathrm{~K}$ and $T=13 \mathrm{~K}$ agree within $90 \%$ for a shift of $\Delta \beta \approx 0.27$. Furthermore, the high calibration uncertainty at $450 \mu \mathrm{m}$ results in a standard deviation error of $\sigma_{\beta} \approx 0.55$ across the B1 clump regardless of the temperature.

SCUBA-2 observations alone cannot constrain both $\beta$ and temperature. If the temperature or $\beta$ across a cloud is roughly constant, then we can probe relative variations using the SCUBA-2 Ratio Case. For regions with known prestellar and protostellar objects like the $\mathrm{B} 1$ complex, it is not ideal to assume constant temperatures or $\beta$ indices, however. For example, Figure 9 reveals a $\sim 6 \mathrm{~K}$ variation in temperature within the 

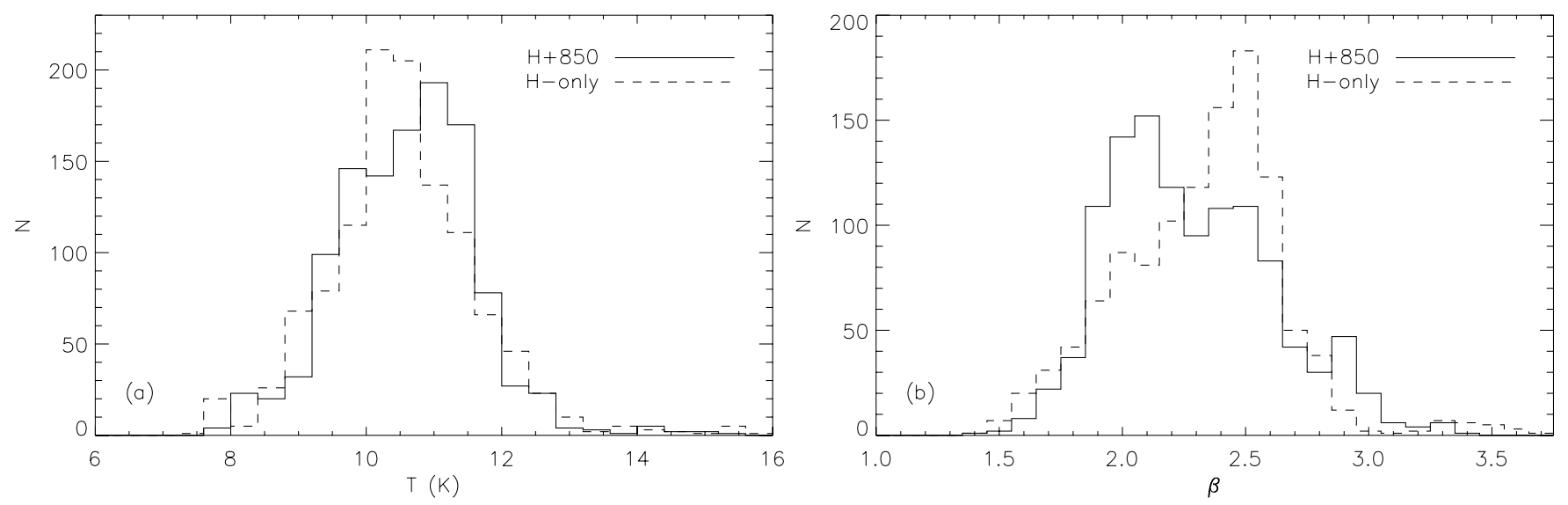

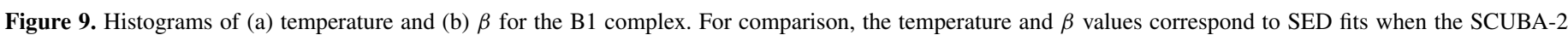

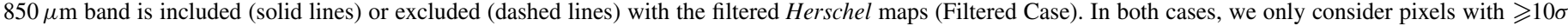
fluxes in all five bands.

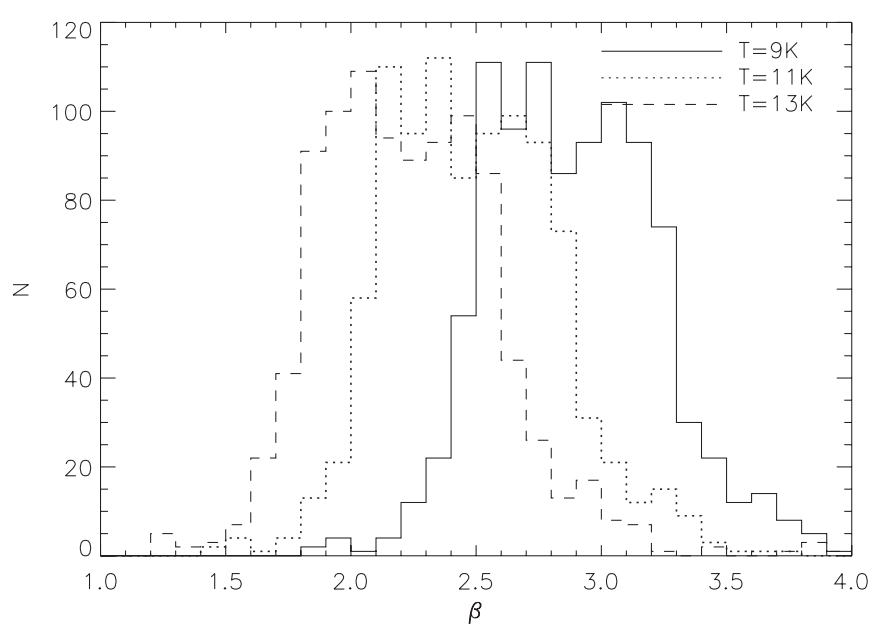

Figure 10. Distributions of $\beta$ for fixed temperatures of $9 \mathrm{~K}, 11 \mathrm{~K}$, and $13 \mathrm{~K}$, and using Equation (2) from the SCUBA-2 Ratio Case.

central $\mathrm{B} 1$ region. A difference of $6 \mathrm{~K}$ in temperature results in a significant uncertainty of $\Delta \beta \approx 0.86$. Similarly, when we fixed $\beta$, we found that the temperature distributions shifted by $\sim 2 \mathrm{~K}$ between $\beta=1.5,2.0$, and 2.5 (see Figure 4). Thus, Figures 4 and 10 illustrate the caution needed for analyses that assume either a fixed $\beta$ or a fixed temperature. In either case, a slight increase or decrease in the fixed parameter can result in significant differences in the determined quantity.

Additionally, in Figure 8, the SEDs show that the observed $450 \mu \mathrm{m}$ fluxes are roughly $15 \%$ greater than those predicted from the SED fits, suggesting that the observed $450 \mu \mathrm{m}$ emission toward these pixels is relatively much brighter than the filtered $500 \mu \mathrm{m}$ emission. Figure 11 shows the fractional flux increase in the observed $450 \mu \mathrm{m}$ data over the $450 \mu \mathrm{m}$ emission predicted from the best-fit SEDs from the Filtered Case. The ratio of observed $450 \mu \mathrm{m}$ emission to predicted $450 \mu \mathrm{m}$ emission peaks around 10\%-15\%. (As described in Appendix B, we believe that this "excess" at $450 \mu \mathrm{m}$ is not due to CO (6-5) contamination.) As a test, we scaled the $450 \mu \mathrm{m}$ emission down by $12.5 \%$ and found that $\beta$ decreased by $\Delta \beta \approx 0.18$ based on a Kolmogorov-Smirnov test. These relative fractions may indicate that the $450 \mu \mathrm{m}$ map does not trace the same material as the $850 \mu \mathrm{m}$ map, e.g., due to different spatial scales filtered out in the reduction or due to temperature variations along the line of sight. Alternatively, these variations could be related to uncertainties in the $450 \mu \mathrm{m}$ fluxes themselves, e.g., from

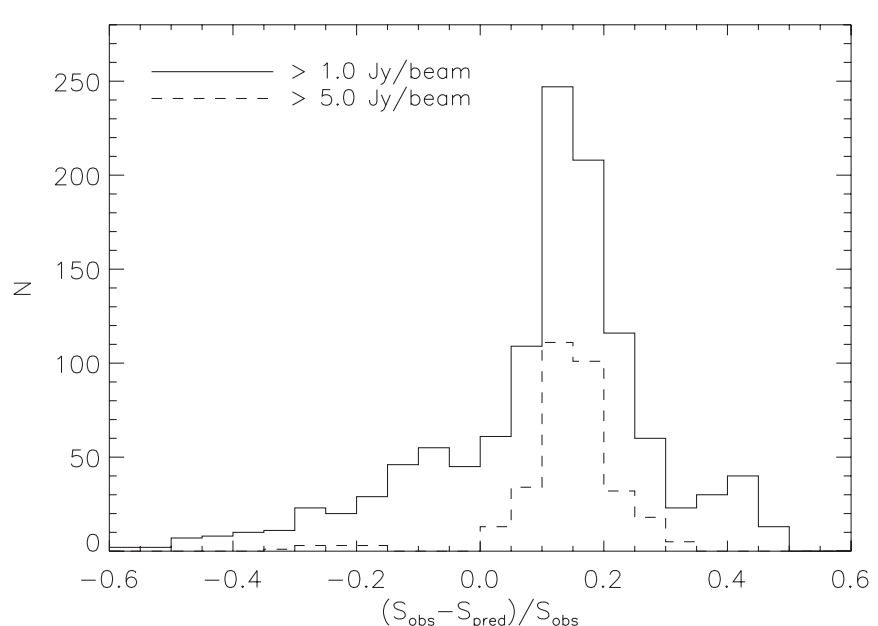

Figure 11. Fractional differences between the observed and predicted $450 \mu \mathrm{m}$ emission for pixels with bright $(>10 \sigma)$ observed $450 \mu \mathrm{m}$ fluxes. These results correspond to the emission predicted from SED fits following the Filtered Case for the $\mathrm{H}+850$ bands.

atmospheric variabilities or the beam pattern. Since we cannot assume a single line-of-sight temperature for the B1 clump, and since the $450 \mu \mathrm{m}$ emission itself is very uncertain, we believe that the SCUBA-2 Ratio Case method is less robust than the Filtered Case method.

\section{2. $\beta$ Using Unfiltered Maps}

For the next two techniques, we determined $\beta$ after attempting to recover the missing large-scale emission filtered out in the SCUBA-2 bands. In Figure 6, the median filtered-out largescale emission at $250 \mu \mathrm{m}$ corresponds to $\sim 1 \mathrm{Jy} \mathrm{beam}^{-1}$ at $18^{\prime \prime}$ resolution (or $\sim 3 \mathrm{Jy}^{\text {beam }}{ }^{-1}$ at $36^{\prime \prime} .3$ resolution). This extended emission peaks toward the central B1 cluster, with additional emission along the southwest filament and to the north. We could naively expect a similar large-scale distribution at $450 \mu \mathrm{m}$ and $850 \mu \mathrm{m}$.

We considered two methods to recover the missing extended emission. The first method, hereafter the Offset Case, seeks to identify the missing large-scale emission at $850 \mu \mathrm{m}$ using a grid of incremental offset values that were added to the observed $850 \mu \mathrm{m}$ map to represent the filtered-out extended emission. We adopted the flux increment that best fit the overall SED to represent the missing emission. For more details, see Appendix D.1. 
The second method, hereafter the Spatial Factor Case, assumes that the observed $450 \mu \mathrm{m}$ and $850 \mu \mathrm{m}$ data correspond to the same spatial scales and that both bands are missing the same fraction of extended emission. For the Spatial Factor Case, we modify the dusty blackbody function in Equation (1) as

$$
I_{v}=\kappa_{v_{0}}\left(v / v_{0}\right)^{\beta} B_{v}(T) \Sigma F_{\mathrm{SF}},
$$

where $F_{\mathrm{SF}}$ is a scaling factor corresponding to the fraction of recovered emission. For the Herschel bands, $F_{\mathrm{SF}}=1$ (i.e., $100 \%$ of the emission is recovered), whereas for the SCUBA-2 bands, $F_{\mathrm{SF}} \leqslant 1$ and we assume that $F_{\mathrm{SF}}$ is identical for both the $450 \mu \mathrm{m}$ band and the $850 \mu \mathrm{m}$ band. For more details, see Appendix D.2.

Recovering the large-scale emission is very uncertain, and we found large uncertainties in our analyses. For the Offset Case, we found uncertainties of $20 \%-50 \%$ for the missing extended emission alone, suggesting that we cannot well constrain the diffuse emission. Thus, the Offset Case cannot be used to constrain $\beta$ from SED fits. For the Spatial Factor Case, the $450 \mu \mathrm{m}$ emission is less certain due to a larger calibration uncertainty of $50 \%$ and as Figure 11 demonstrates, the observed $450 \mu \mathrm{m}$ emission tends to be $10 \%-15 \%$ brighter than the predicted (filtered) emission. Since the scaling factor is very dependent on both the $450 \mu \mathrm{m}$ and $850 \mu \mathrm{m}$ fluxes, the large flux uncertainties at $450 \mu \mathrm{m}$ will greatly affect the final results. Finally, based on the filtered Herschel maps (see Section 4.1), the fraction of emission lost in the filtering appears to vary considerably with wavelength. Therefore, $F_{\mathrm{SF}}$ may actually vary somewhat between the $450 \mu \mathrm{m}$ and $850 \mu \mathrm{m}$ bands. For the $\beta$ results using these two techniques, see Appendices D.1 and D.2.

\subsection{Comparison of the Techniques}

The Filtered Case is the most robust technique to combine SCUBA-2 data with PACS and SPIRE data. For the Filtered Case, we have demonstrated that the $850 \mu \mathrm{m}$ band makes a significant improvement to the uncertainties associated with SED fitting (see Figure 7) and impacts the absolute value of $\beta$. For example, we determined that the $\mathrm{H}$-only results generally underestimated $\beta$ toward the dense cores and overestimated $\beta$ along the filament. These observations are in agreement with a recent study by Kelly et al. (2012), which suggests that SED fitting through the minimization of $\chi^{2}$ may be biased toward lower $\beta$ values for prestellar cores.

Using the Filtered Case technique, we find $\beta \approx 1.6-2$ for the dense cores with the lowest values toward the B1-c core. In addition, we find $\beta \approx 2$ along the filament and $\beta \gtrsim 2$ toward the moderately dense clump material. Similarly, Friesen et al. (2005) found $\beta \approx 1.3-2.1$ using a Fourier Transform Spectrograph at $350 \mathrm{GHz}$ to observe several hot molecular cores. Other studies, however, have identified larger $\beta$ values toward dense cores. For example, Schnee et al. (2010) found $\beta \approx 1.7-2.7$ toward a starless core using mid-infrared and (sub)millimeter continuum observations, and Shirley et al. (2011) found $\beta \approx 2.2-2.6$ toward a young protostellar core using radiative transfer models with SCUBA flux ratios and near-infrared color excesses. Additionally, Arab et al. (2012) found $\beta \sim 2$ values toward the cooler $(\sim 40 \mathrm{~K})$, denser regions of the Orion bar and $\beta \sim 1.1$ toward the warmer $(\sim 70 \mathrm{~K})$ regions using Herschel and Spitzer observations. Thus, a larger study of dense cores and their environments with the same set of observations is necessary to understand these differences in $\beta$.

With the remaining three techniques, $\beta$ is unclear. For the SCUBA-2 Ratio Case (Section 4.1), a minor variation in

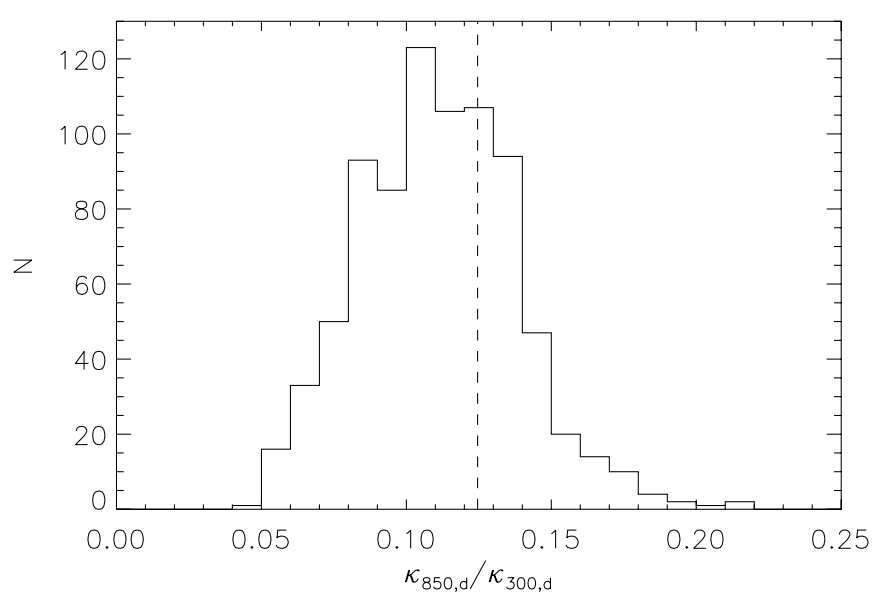

Figure 12. Distribution of the dust opacity at $850 \mu \mathrm{m}$ relative to the dust opacity at $300 \mu \mathrm{m}$ using the Filtered Case. The dashed line shows the relative dust opacities for $\beta=2$.

temperature greatly affects the measure of $\beta$; for the Offset Case (Section 4.2 and Appendix D.1), the Herschel calibration uncertainties result in very uncertain $850 \mu \mathrm{m}$ offsets; and for the Spatial Factor Case (Section 4.2 and Appendix D.2), the $450 \mu \mathrm{m}$ band is very uncertain, and thus causes a wide range of plausible scale factors. Additionally, it is not clear that the $450 \mu \mathrm{m}$ and $850 \mu \mathrm{m}$ observations trace the same scales. Both the SCUBA-2 Ratio Case and the Spatial Factor Case require the $450 \mu \mathrm{m}$ band.

In Section 5, we discuss the results for the Filtered Case only. For comparison, we will consider both the H-only and $\mathrm{H}+850$ results.

\section{DISCUSSION}

\subsection{Submillimeter Dust Opacity and Dust Masses}

We are interested in comparing the dust opacity between the dense cores and the ambient clump material. In Equation (1), we assumed that the dust opacity follows a power-law distribution for a reference opacity, $\kappa_{\nu_{0}}$, at $1000 \mathrm{GHz}(300 \mu \mathrm{m})$. Ideally, we would want to fit $\kappa_{\nu_{0}}$ as well as $\beta$. According to Ossenkopf \& Henning (1994), the dust opacity at $300 \mu \mathrm{m}$ increases by a factor of $\lesssim 2$ for coagulated dust grains with icy mantles (this effect is more significant for grains without icy mantles). More recently, Martin et al. (2012) and Roy et al. (2013) used submillimeter dust emission and near-infrared extinction maps to constrain the submillimeter dust and gas opacity at $250 \mu \mathrm{m}$ for $\beta=1.8$. Both studies found a steady increase in dust opacity (by factors of 2-4) toward colder temperatures, suggesting that the dust opacity should not be fixed at $300 \mu \mathrm{m}$.

Unfortunately, we cannot separate out the degeneracy of $\kappa_{v_{0}}$ with column density in B1 at this time. Although $\kappa_{\nu_{0}}$ can be constrained by independent measurements of column density, we are interested in changes to the dust over small scales (i.e., between dense cores), and as such we cannot utilize extinction maps (at $\sim 2^{\prime}$ resolutions) as done by Martin et al. (2012) and Roy et al. (2013). Therefore, while we cannot measure absolute values of $\kappa_{v}$ with our observations, we can probe the variations in $\kappa_{v}$ due to our differences in $\beta$. Thus, we focus on the relative variations in $\kappa_{v}$ with the $\mathrm{H}+850$ data and the significance of assuming $\beta=2$, as with most Herschel analyses of molecular clouds.

Figure 12 shows the dust opacity at $850 \mu \mathrm{m}$ relative to the opacity at $300 \mu \mathrm{m}$ for the Filtered Case. This measure does not assume any prior knowledge of the dust opacity at either $300 \mu \mathrm{m}$ 

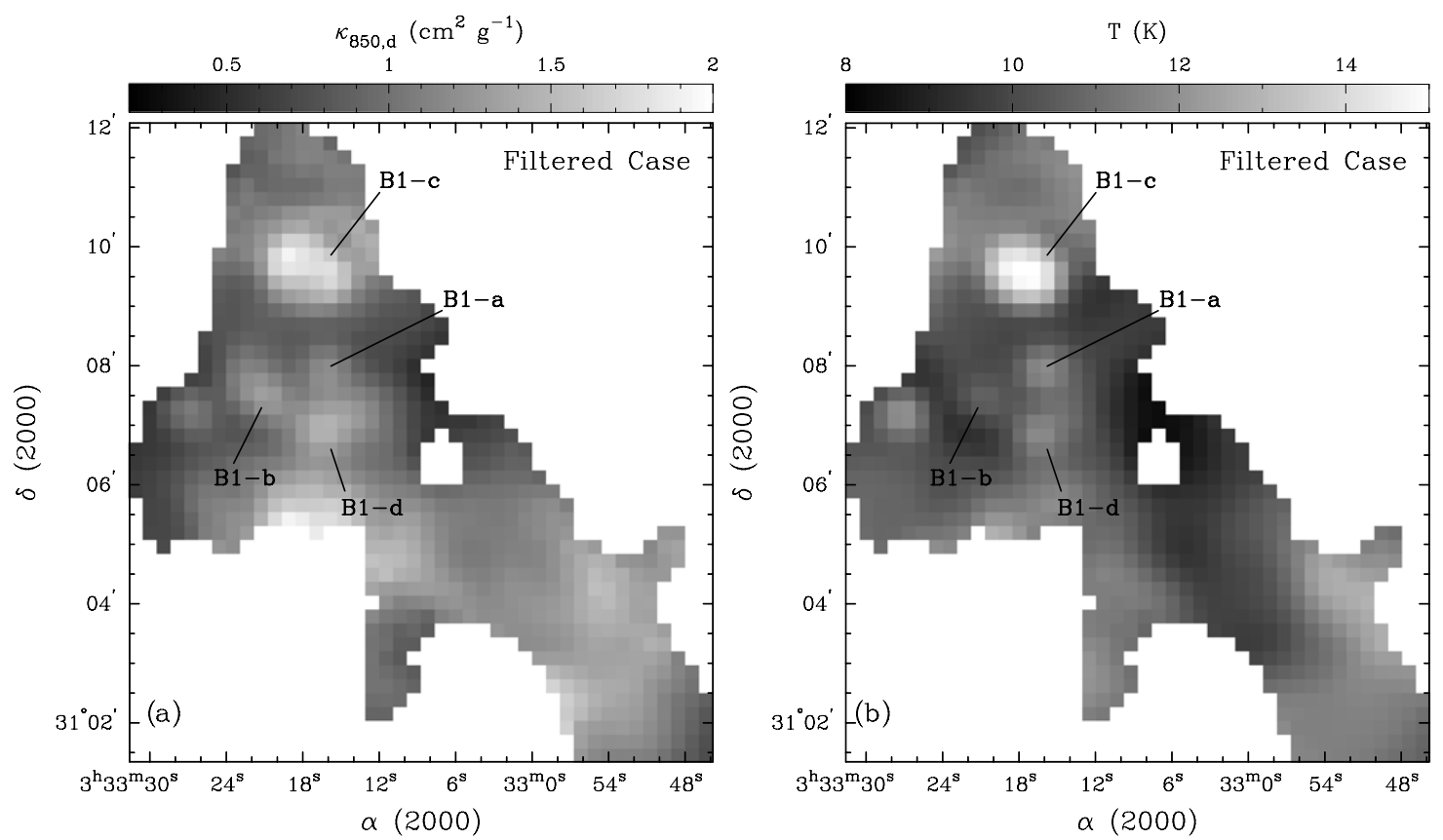

Figure 13. Results from the Filtered Case showing (a) the dust opacity at $850 \mu \mathrm{m}$ and (b) the dust temperature. The dust opacity map assumes $\kappa_{v, \mathrm{~d}}=10 \mathrm{~cm}^{2} \mathrm{~g}^{-1}$ at $300 \mu \mathrm{m}$ and that all variations in $\beta$ manifest themselves as changes in $\kappa_{\nu, d}$ at $850 \mu \mathrm{m}$.

or $850 \mu \mathrm{m}$. Thus, Figure 12 demonstrates that the relative dust opacity at $850 \mu \mathrm{m}$ increases by a factor of $2-3$ between the moderately dense clump material and the dense B1-c core, which suggests that the grains are evolving. Dust coagulation alone can increase the submillimeter dust opacity by factors of $4-5$ at $850 \mu \mathrm{m}$, though this increase is enhanced for bare dust grains and suppressed with surface ices (Ossenkopf \& Henning 1994). Alternatively, Ossenkopf \& Henning (1994) noted that moderately elliptical dust grains or higher abundances of metal compounds can increase submillimeter dust opacities at $850 \mu \mathrm{m}$ by factors of a few. Without an independent measure of the dust opacity or column density, however, we cannot directly measure the absolute variations in dust opacity. Thus, further observations of the clump chemistry and dust are necessary to help determine the causes of these opacity variations.

To measure column densities and masses, however, we must assume a dust opacity law. Thus, we adopted a dust opacity per unit dust mass of $\kappa_{v, d}=10(\mathrm{v} / 1 \mathrm{THz})^{\beta} \mathrm{cm}^{2} \mathrm{~g}^{-1}$, following our assumed dust and gas opacity, $\kappa_{\nu_{0}}=0.1 \mathrm{~cm}^{2} \mathrm{~g}^{-1}$ at $1 \mathrm{THz}(300 \mu \mathrm{m})$, and a dust-to-gas mass ratio of 100 . Figure 13 shows the resulting map for the dust opacity at $850 \mu \mathrm{m}$ and the distribution of best-fit temperatures from the Filtered Case using the $\mathrm{H}+850$ bands. Based on our $1 \sigma$ uncertainties for $\beta$, we find that the uncertainties on $\kappa_{v, d}$ are $10 \%-30 \%$, where the largest errors are found toward the compact cores. These results are only valid if the dust opacity is kept fixed at $300 \mu \mathrm{m}$. From the temperature map, B1-a, B1-c, and B1-d show internal heating from protostellar sources. B1-b contains two first hydrostatic core candidates (see Pezzuto et al. 2012), and we see an indication of moderate heating toward these cores (at 36" resolutions, we cannot separate the two components). The uncertainties on the dust temperatures are predominantly $\sigma_{T}<1.5 \mathrm{~K}$, with the largest errors toward B1-c. The dust temperature is $\sim 10.5 \mathrm{~K}$ and $\sim 12 \mathrm{~K}$ for $\mathrm{B} 1-\mathrm{b}$ and $\mathrm{B} 1-\mathrm{d}$, respectively, which are similar to the $\mathrm{NH}_{3}$-derived kinetic gas temperatures of $\sim 11.5 \mathrm{~K}$ reported in Rosolowsky et al. (2008). The kinetic gas temperature of B1-c, however, is $\sim 12.4 \mathrm{~K}$ (Rosolowsky et al. 2008), which is lower than the observed dust
Table 2

Core Mass Estimations ${ }^{\mathrm{a}}$

\begin{tabular}{lccc}
\hline \hline Core & B1-b & B1-c & B1-d \\
\hline $\mathrm{H}+850$ & $15 M_{\odot}$ & $5 M_{\odot}$ & $9 M_{\odot}$ \\
H-only & $13 M_{\odot}$ & $6 M_{\odot}$ & $9 M_{\odot}$ \\
\hline
\end{tabular}

Notes.

a Masses measured from the column density maps using the Filtered Case and assuming $\kappa_{v}=0.1 \mathrm{~cm}^{2} \mathrm{~g}^{-1}$ at $300 \mu \mathrm{m}$. For the $\mathrm{H}+850$ case, the fixed dust opacity at $300 \mu \mathrm{m}$ is the greatest source of uncertainties, so these masses are only accurate within a factor of $\sim 2$. For the $\mathrm{H}$-only case, forcing $\beta=2$ increases the uncertainties in the masses by, at most, an additional factor of two.

${ }^{\mathrm{b}}$ Masses measured from filtered H-only maps assuming $\beta=2$.

temperatures of $\sim 15 \mathrm{~K}$. This difference in temperature suggests that the $\mathrm{NH}_{3}$ gas is tracing a different region of the core than the dust (e.g., see Matthews et al. 2006).

Figure 14 compares the $N\left(\mathrm{H}_{2}\right)$ column densities measured toward the $\mathrm{B} 1$ cores for (1) the $\mathrm{H}+850$ bands and (2) the $\mathrm{H}$-only bands with $\beta=2$ (i.e., using the method employed by most Herschel GBS papers). The gas column density is determined following Equation (1) and assuming $\kappa_{\nu}=0.1 \mathrm{~cm}^{2} \mathrm{~g}^{-1}$ at $300 \mu \mathrm{m}\left(\kappa_{v, \mathrm{~d}}=10 \mathrm{~cm}^{2} \mathrm{~g}^{-1}\right.$ for a gas-to-dust ratio of 100$)$. Table 2 lists the total (gas and dust) mass estimates for the B1-b, B1-c, and B1-d cores using these column density maps. For simplicity, we calculated the masses associated with $5 \times 5$ pixel boxes around the cores. For the Herschel-only data with $\beta=2$, we underestimate the columns of mass toward B1-b by $\sim 10 \%$ and overestimate the columns of mass toward B1-c by $\sim 30 \%$ (the masses toward B1-d agree within 1\%). Since we find $\beta \approx 2$ for much of the B1 clump (see Figure 9), assuming $\beta=2$ with Herschel-only data appears to provide a decent first look at the mass distribution of dense cores and the column density of extended cloud emission. Nevertheless, for regions that deviate from $\beta=2$, such as B1-c $(\beta \approx 1.6-1.7)$, we find that the SED fitting to the Herschel-only bands with $\beta=2$ yields more significant mass uncertainties $(\sim 30 \%)$. Therefore, accurate core masses or column densities require direct measurements of $\beta$. 

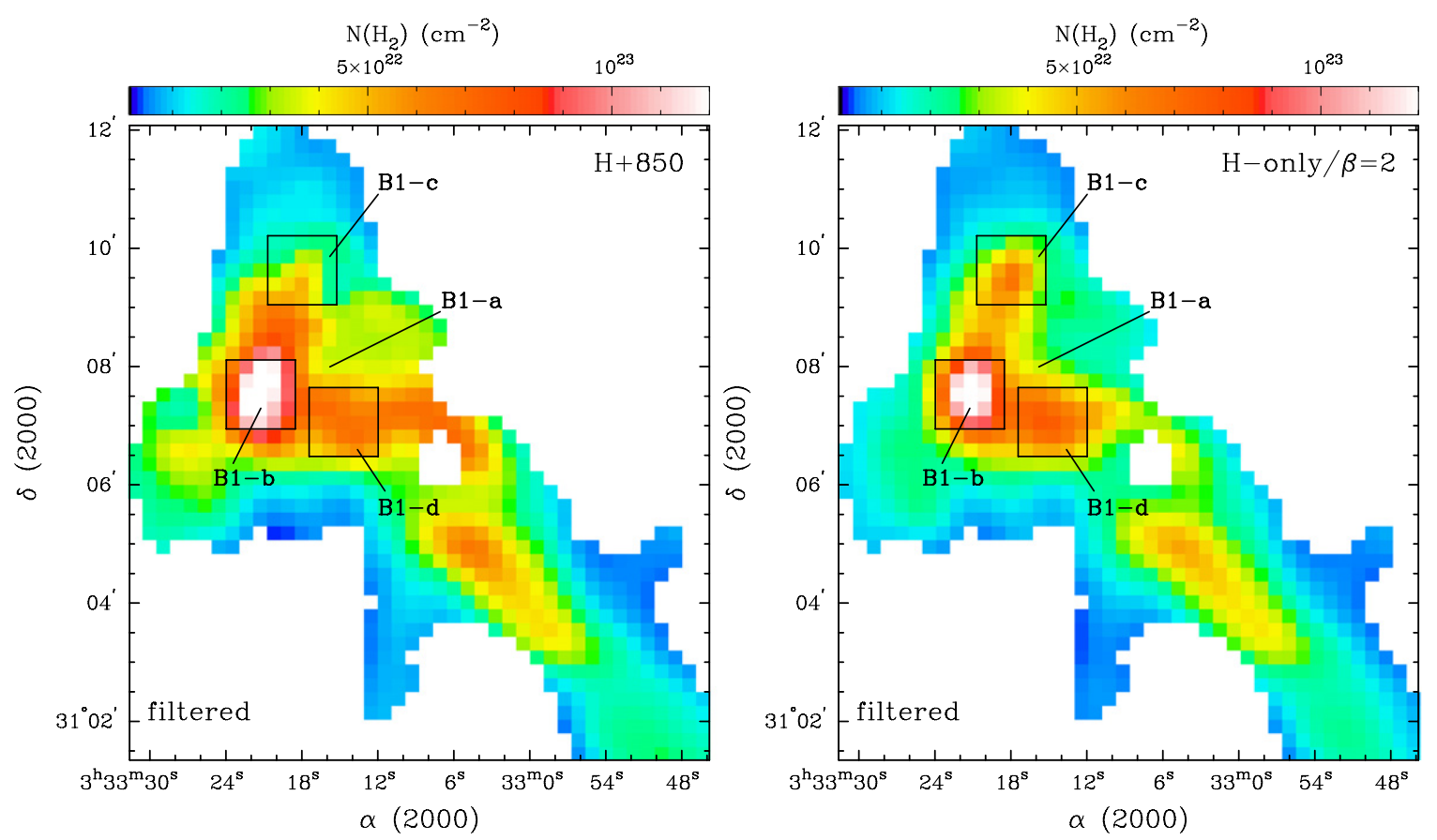

Figure 14. Column density maps using the filtered Herschel data. For panel (a), we used the $\mathrm{H}+850$ bands and the $\beta$ and temperature results from the Filtered Case, and for panel (b) we used the H-only bands and fixed $\beta=2$. The black boxes correspond to our mass measurements.

For example, assuming $\beta=2$ could affect our ability to classify cores as dynamically stable or unstable, to properly compare the core mass function to the stellar initial mass function, and to calculate relative (to $\mathrm{H}_{2}$ ) abundances of molecules.

\subsection{Temperature and $\beta$}

In the literature, the dust emissivity index is often assumed to be $\beta=2$ based on studies of the dielectric functions of graphite and silicate dust grains (e.g., Draine \& Lee 1984). Deviations from $\beta=2$ can arise from processes such as grain growth and the accumulation of icy mantles. For example, $\beta<2$ has been attributed to the coagulation of bare dust grains (e.g., Schwartz 1982; Ossenkopf \& Henning 1994; Lis et al. 1998) and Beckwith \& Sargent (1991) attributed very low $\beta$ values $(\sim 1)$ toward protostellar disks to grain growth. Large values of $\beta$ (e.g., >2), however, are harder to interpret. Thick icy mantles can steepen the dust opacity slope at submillimeter wavelengths, but this appears to be a lesser effect than dust coagulation (e.g., Ossenkopf \& Henning 1994). Unfortunately, most dust models do not predict dust opacities with $\beta>2$ (Shirley et al. 2011). Meny et al. (2007) found that $\beta>2$ may arise due to complex interactions between electromagnetic radiation and the disordered mass and charge structures of amorphous dust grains. Thus, $\beta$ highly reflects the dust grain properties, and it is important to understand the relationship between $\beta$ and the surrounding environment.

Nevertheless, the relationship between $\beta$ and temperature is not well understood. Many studies that fit submillimeter SEDs to determine $\beta$ use the minimization of $\chi^{2}$ technique as employed here. Several recent studies, however, have suggested that the minimization of $\chi^{2}$ produces unclear results due to the degeneracy between $\beta$ and temperature, especially toward the SED peak (e.g., Veneziani et al. 2010; Kelly et al. 2012). Since the Herschel bands span the SED peak for cold star-forming regions, the method of SED fitting becomes significant. In particular, Kelly et al. (2012) demonstrated that the $\chi^{2}$ minimization tech- nique gives different temperature and emissivity values than a more detailed Bayesian approach. In addition to the fitting technique, an artificial anti-correlation between $\beta$ and temperature can also be introduced by instrumental errors (e.g., Shetty et al. 2009a) or temperature variations along the line of sight (e.g., Shetty et al. 2009b; Schnee et al. 2010).

Despite these uncertainties, several large studies have found strong inverse relationships between $\beta$ and temperature over large areas and a wide range of temperatures. These studies include the PRONAOS experiment (e.g., Dupac et al. 2003), the Herschel Hi-Gal project (e.g., Paradis et al. 2010), and early science results from Planck (e.g., Planck Collaboration et al. 2011). In particular, Paradis et al. (2010) employed two techniques to their SED fitting, a minimization of $\chi^{2}$ method and a maximum likelihood algorithm. They found an inverse $\beta$-temperature relationship with both methods.

In Figure 7, we find that the uncertainties on our values of $\beta$ and temperature are such that small degeneracies may persist between any two values determined, and so any anticorrelation seen between $\beta$ and temperature may be artificial. Thus, despite our improved constraints to determine $\beta$ with the $850 \mu \mathrm{m}$ data, the SED fits may still retain some degeneracies. From our data, we believe that the causes of any degeneracy are likely dominated more by our use of the $\chi^{2}$ technique rather than the noise or single-component line-of-sight-temperature assumption. For example, we only selected very bright emission, so issues with noise will be negligible. Additionally, our analysis used filtered observations, and thus the line-of-sight temperature is less affected by warm diffuse material. The protostellar cores, however, will have warm central objects and cool outer envelopes, and thus assuming a single line-of-sight temperature toward these objects may induce a stronger degeneracy between $\beta$ and temperature. Nevertheless, with only five bands in the submillimeter domain, we cannot apply a multiple temperature component fit. Thus, with our analyses, we cannot make any robust conclusions regarding any physical $\beta$-temperature relationship. 


\subsection{The Addition of SCUBA-2 Data}

Figure 7 demonstrates that the SED fits are much improved with the inclusion of $850 \mu \mathrm{m}$ observations since the Herschel data alone are unable to constrain $\beta$ for cold star-forming regions. Since the $850 \mu \mathrm{m}$ emission is more dependent on $\beta$ (see Figure 5), the SCUBA-2 observations have a significant influence on the best-fit SEDs and the determined values of $\beta$. Therefore, this work demonstrates that the SCUBA-2 $850 \mu \mathrm{m}$ data (or equivalent long-wavelength observations) are essential for constraining SED fits.

The SCUBA-2 $450 \mu \mathrm{m}$ band has superior resolution $\left(\sim 9^{\prime \prime}\right)$ to the Herschel data at $160-500 \mu \mathrm{m}$ presented here (see Figure 2), which is necessary for deblending compact sources to obtain more accurate flux measurements. Additionally, with the $450 \mu \mathrm{m}$ band, we can measure dust properties at higher resolutions. For example, in this paper, we convolved our data to a common resolution of 36.3 (corresponding to the $500 \mu \mathrm{m}$ beam), but with the SCUBA-2 $450 \mu \mathrm{m}$ band, we could exclude the SPIRE $500 \mu \mathrm{m}$ band and convolve to a common resolution of $25^{\prime \prime}$ (the $350 \mu \mathrm{m}$ beam), and find the dust temperature, opacity, and column density over smaller scales, thereby probing variations across cores themselves.

Unfortunately, the $450 \mu \mathrm{m}$ emission has significant uncertainties. For example, in Figures 8 and 11, we see that the observed $450 \mu \mathrm{m}$ emission is roughly $10 \%-15 \%$ brighter than the predicted emission. Additionally, the $450 \mu \mathrm{m}$ band is more susceptible to atmospheric variability. Therefore, we do not use the $450 \mu \mathrm{m}$ band at this time, but note that with better calibrations and atmospheric modeling, the $450 \mu \mathrm{m}$ band will become more robust and higher resolution analyses will become possible.

\subsection{High-resolution Extinction Maps}

In Section 5.1, we assumed a fixed dust opacity at $300 \mu \mathrm{m}$ to measure column densities and masses. To circumvent fixing $\kappa_{v}(300 \mu \mathrm{m})$, we need to measure the dust opacity or column density through an independent method, such as from extinction maps. Since we are interested in measuring changes in dust opacity over small scales, we need high-resolution $\left(<1^{\prime}\right)$ extinction maps. Such observations would greatly improve the analysis in this paper.

Typically, extinction maps have resolutions of $\gtrsim 2^{\prime}$, which are too coarse to probe changes in dust opacity between dense cores and the ambient clump. Nevertheless, high-resolution $\left(\sim 20^{\prime \prime}\right)$ extinction maps are possible with deep near-infrared observations (e.g., Román-Zúñiga et al. 2010). Therefore, future studies of dust opacity using Herschel and JCMT observations will greatly benefit from such high-resolution extinction mapping.

\section{SUMMARY}

We have presented four unique methods to measure the dust emissivity index, $\beta$, from a combination of Herschel PACS+SPIRE and SCUBA-2 observations. Of these methods, the Filtered Case, where the Herschel maps are filtered using the SCUBA-2 reduction pipeline and $\beta$ is determined from SED fitting to the filtered Herschel+850 bands, is the most robust. Our main goal was to examine whether or not the SCUBA-2 $850 \mu \mathrm{m}$ band made significant improvements to the SED fits and the relative differences in $\beta$ (or $\kappa_{\nu, d}$ ) that arise from those fits. We summarize our main findings as follows.

1. The Herschel-only data do not constrain the SED fits well enough to allow simultaneous fitting of $\beta$ and tempera- ture toward cold clouds. In Figure 7, we demonstrated that long-wavelength observations are necessary to improve SED fitting to the Herschel bands, provided that the long-wavelength observations have relatively low flux errors. The $850 \mu \mathrm{m}$ SCUBA-2 band improves our ability to constrain $\beta$ by a factor of $\sim 2$ and our ability to determine temperature by $\sim 40 \%$. Additionally, we find significant variations in $\beta$ when the $850 \mu \mathrm{m}$ data are included, i.e., we find $\beta \sim 1.6$ toward the $\mathrm{B} 1-\mathrm{c}$ core and $\beta \sim 2.0$ toward the filament.

2. Based on our SED fitting, we find that the dust opacity at $850 \mu \mathrm{m}$ varies by factors of $2-3$ relative to the dust opacity at $300 \mu \mathrm{m}$, with the highest opacities toward the dense cores, particularly B1-c. Therefore, the B1-c core may represent a region where the dust grains have coagulated with some icy mantles, have moderate elliptical shapes, or have higher abundances of metal compounds (Ossenkopf \& Henning 1994). Our observations cannot constrain the source of the opacity variations. Future studies with highresolution extinction mapping are needed to probe the dust opacity in more detail.

3 . With our analysis, we cannot make robust conclusions about the $\beta$-temperature relationship. We found that both $\beta$ and temperature are constrained better with the inclusion of $850 \mu \mathrm{m}$ observations (see Figure 7), yet the degeneracy between these parameters is not entirely removed. While this degeneracy likely arises from using the minimization of $\chi^{2}$ technique to fit the SEDs, there can be additional degeneracies due to assuming a single-component line-ofsight temperature.

4. Assuming a fixed $\beta=2$ value with the Herschel bands alone affects the measured best-fit temperature by $\lesssim 2 \mathrm{~K}$ if $\beta=1.5$ or $\beta=2.5$. We found that the core masses as measured with the Herschel-only bands and with $\beta=2$ varied by $\lesssim 30 \%$ compared to the results using Herschel +850 bands and the same assumptions for the dust opacity $\left(\kappa_{\nu_{0}}=0.1 \mathrm{~cm}^{2} \mathrm{~g}^{-1}\right.$ at $\left.300 \mu \mathrm{m}\right)$. Thus, the Herschel-only bands provide a decent first look at the core masses for an assumed value of $\beta=2$. Nevertheless, to obtain more accurate core masses, i.e., to measure the dynamical stability of dense cores or the core mass function, long-wavelength data and high-resolution extinction maps are necessary.

This analysis of the B1 clump with PACS+SPIRE+SCUBA-2 represents the first of many future analyses with these instruments. The SCUBA-2 observations are an excellent complement to the Herschel bands, providing a much stronger constraint to SED fitting and derivations of $\beta$. Herschel and long-wavelength observations such as those from SCUBA-2 have only recently provided the wavelength and spatial coverage necessary to make these measurements. Future analyses using high-resolution extinction maps are also important to probe the changes in $\kappa_{v, d}$ with environment.

This work was possible with funding from the Natural Sciences and Engineering Research Council of Canada (NSERC) Canadian Graduate Student award. We acknowledge the support by the Canadian Space Agency (CSA) via a Space Science Enhancement Program grant, the National Science and Engineering Research Council (NSERC) via a Discovery grant, and the National Research Council of Canada (NRC). We thank the anonymous referee for detailed comments that greatly improved the clarity of this paper. We thank E. Chapin, 
J. Dempsey, P. Friberg, A. Gibb, and S. Schnee for their invaluable advice and assistance throughout this project. We thank B. Ali, B. Altieri, and M. Griffin for useful discussions about PACS and SPIRE calibration. The JCMT is operated by the Joint Astronomy Centre (JAC) on behalf of the Science and Technology Facilities Council (STFC) of the United Kingdom, the National Research Council (NRC) of Canada, and the Netherlands Organisation for Scientific Research. The SCUBA-2 project is funded by the UK Science and Technology Facilities Council (STFC), the JCMT Development Fund and the Canadian Foundation for Innovation (CFI). Herschel is an ESA space observatory with science instruments provided by European-led Principal Investigator consortia and with important participation from NASA. PACS has been developed by a consortium of institutes led by MPE (Germany) and including UVIE (Austria); KU Leuven, CSL, IMEC (Belgium); CEA, LAM (France); MPIA (Germany); INAF-IFSI/OAA/OAP/ OAT, LENS, SISSA (Italy); IAC (Spain). This development has been supported by the funding agencies BMVIT (Austria), ESAPRODEX (Belgium), CEA/CNES (France), DLR (Germany), ASI/INAF (Italy), and CICYT/MCYT (Spain). SPIRE has been developed by a consortium of institutes led by Cardiff University (UK) and including Univ. Lethbridge (Canada); NAOC (China); CEA, LAM (France); IFSI, Univ. Padua (Italy); IAC (Spain); Stockholm Observatory (Sweden); Imperial College London, RAL, UCL-MSSL, UKATC, Univ. Sussex (UK); and Caltech, JPL, NHSC, Univ. Colorado (USA). This development has been supported by national funding agencies: CSA (Canada); NAOC (China); CEA, CNES, CNRS (France); ASI (Italy); MCINN (Spain); SNSB (Sweden); STFC (UK); and NASA (USA).

\section{APPENDIX A}

\section{SCUBA-2 FILTERING}

Since SCUBA-2 is a ground-based detector, we filter out large-scale astronomical emission in the process of removing the emission from a bright and variable atmosphere. Thus, SCUBA-2 is sensitive only to spatial scales where emission is fully recovered. To determine the largest such spatial scale, we introduced artificial Gaussian sources of various sizes ranging from $1^{\prime}$ to 7.5 FWHM to the original SCUBA-2 data, and then ran these modified maps through the pipeline. Since the SCUBA-2 pipeline determines which map pixel is covered by which bolometer at any given time, the extra signal corresponding to the Gaussian sources is simply added to the bolometer time series. Following our reduction process from Section 2.2, the smaller Gaussians were fully recovered, e.g., Gaussians of $1^{\prime}$ or 2'.5 sizes. Larger Gaussians, however, were only partially recovered or fully filtered out. For example, peak fluxes for $\sim 5^{\prime}$ Gaussians were only $70 \%$ recovered. Based on these tests, the SCUBA-2 observations appear to fully recover objects on size scales $\lesssim 2.5$.

Conversely, Herschel is a space-based observatory, and thus, the PACS+SPIRE data are unaffected by atmospheric emission. For our HARP CO (3-2) line observations, we used an emissionfree off position to remove the atmospheric contributions. Thus, both PACS+SPIRE and HARP observations are unfiltered, and such data cannot be directly compared to the SCUBA-2 maps. One simple solution is to filter out these large-scale structures, such that the Herschel continuum and HARP line observations are sensitive to the same spatial scales as the SCUBA-2 data.

We filtered out the large-scale emission from the $\mathrm{CO}(3-2)$ and Herschel maps using the SCUBA-2 reduction pipeline in the same manner as the artificial Gaussian sources. We created "artificial maps" by adding the unfiltered CO (3-2) integrated intensity map (in $\mathrm{Jy} \mathrm{beam}^{-1}$ ) or the Herschel data to the SCUBA-2 bolometer time series by adjusting the original signal observed at every bolometer to include the emission observed by HARP, PACS, or SPIRE. Using these artificial signals, we repeated the SCUBA-2 reduction as given in Section 2.2 four times, once for each of the four Grade 1 observations. Finally, we combined the products into a single mosaic. Since this mosaic also includes the original $850 \mu \mathrm{m}$ data, we subtracted out the reduced $850 \mu \mathrm{m}$ map to leave behind the filtered version of the other data (see Figure 6).

\section{APPENDIX B}

\section{CO LINE CONTAMINATION}

The CO (3-2) line transition coincides with the $850 \mu \mathrm{m}$ SCUBA-2 band, and thus will contaminate the continuum emission. Significant CO (3-2) contamination is mostly identified toward outflows and highly energetic regions such as $\mathrm{H}$ II regions, where the contamination levels can dominate $(>50 \%)$ the total continuum emission (e.g., Davis et al. 2000; Tothill et al. 2002; Drabek et al. 2012). Although B1 is a low-mass star-forming complex, and such regions generally contain weaker CO (3-2) line emission (Drabek et al. 2012), this clump contains several protostellar sources driving outflows (Bally et al. 2008).

To evaluate the $\mathrm{CO}(3-2)$ contamination, we determined the contribution from the line flux over the entire SCUBA-2 $850 \mu \mathrm{m}$ passband. Following Drabek et al. (2012), the line flux at frequency $v_{0}$ across the continuum band is

$$
\frac{F_{\nu_{0}}}{\text { mJy beam }^{-1}}=\frac{2 k v_{0}^{3}}{c^{3}} \frac{g_{\nu_{0}, \text { line }}}{\int g_{v, \text { cont }} d v} \Omega \int T_{\mathrm{MB}} d v
$$

where $k$ is the Boltzmann constant, $c$ is the speed of light, $\Omega$ is the telescope beam, $T_{\mathrm{MB}}$ is the main beam temperature of the line, $g_{v_{0} \text {, line }}$ is the transmission of the submillimeter filter at the frequency of the molecular line $\left(v_{0}\right)$, and $g_{v, \text { cont }}$ is the transmission profile of the entire submillimeter filter. Drabek et al. (2012) adopted a "conversion factor" as

$$
\frac{C}{\mathrm{mJy} \mathrm{beam}^{-1}\left(\mathrm{~K} \mathrm{~km} \mathrm{~s}^{-1}\right)^{-1}}=\frac{2 k v_{0}^{3}}{c^{3}} \frac{g_{\nu_{0}, \text { line }}}{\int g_{\nu, \text { cont }} d \nu} \Omega .
$$

The SCUBA-2 filter profile, $g_{v, \text { cont }}$, varies significantly with atmospheric conditions. For example, the filter response is best for dry conditions $\left(\tau_{225}<0.05\right)$ and can drop by more than $50 \%$ in wet conditions $\left(\tau_{225}>0.12\right)$. We used the S2SV observations made in very dry conditions, $\tau_{225}<0.05$, resulting in $C \approx 0.63 \mathrm{mJy} \mathrm{beam}^{-1}\left(\mathrm{~K} \mathrm{~km} \mathrm{~s}^{-1}\right)^{-1}$ (Drabek et al. 2012). This factor, however, assumes that the beam of SCUBA-2 at $850 \mu \mathrm{m}$ is $13^{\prime \prime} .8$. We have adopted here an effective beam of $14^{\prime \prime} .2$ (see Section 2.2), which considers the larger error beam. Thus, our conversion factor should be modified by $\Omega_{\text {eff }} / \Omega=1.06$. Therefore, we use

$$
C=0.67 \mathrm{mJy} \mathrm{beam}^{-1}\left(\mathrm{~K} \mathrm{~km} \mathrm{~s}^{-1}\right)^{-1} .
$$

In addition to $\mathrm{CO}$ (3-2) line contamination, other (weaker) molecular line emission will contaminate the continuum bands (Drabek et al. 2012). For example, CO (6-5) at $691.473 \mathrm{GHz}$ coincides with the $450 \mu \mathrm{m} \mathrm{SCUBA}-2$ band and CO (7-6) at $806.652 \mathrm{GHz}$ coincides with the SPIRE $350 \mu \mathrm{m}$ band. Nevertheless, Drabek et al. demonstrated that such high excitation 

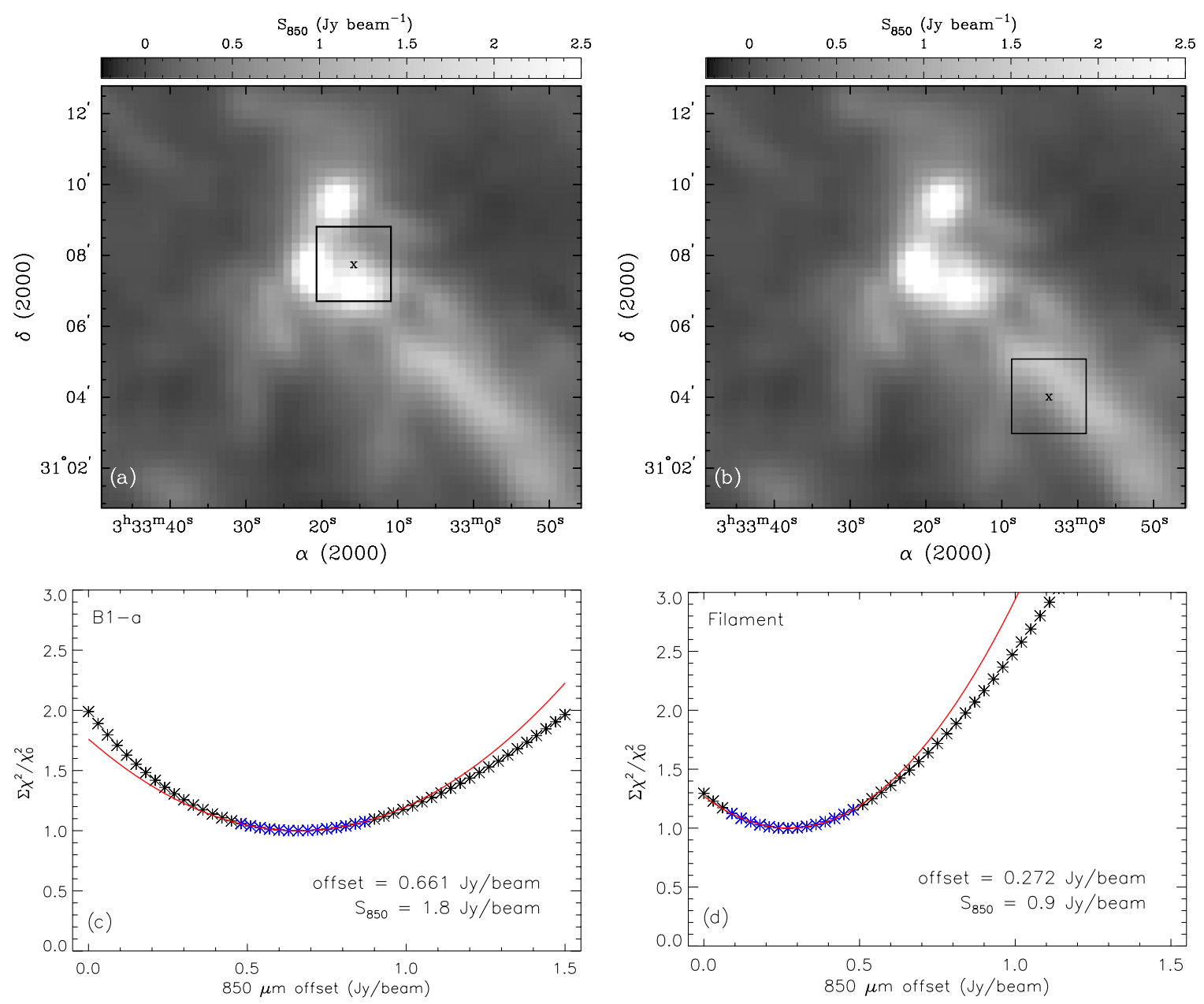

Figure 15. Technique to measure the missing $850 \mu \mathrm{m}$ emission. Top: two examples of $9 \times 9$ pixel subregions centered on (a) B1-a and (b) the filament. For a given subregion, the SEDs of each pixel are fit for a range of $850 \mu \mathrm{m}$ offset values from 0 to $1.5 \mathrm{Jy} \mathrm{beam}^{-1}$. Then, for each offset estimate, we sum the individual $\chi^{2}$ measurements from every best-fit SED in the subregion. Bottom: the relative total $\chi^{2}$ measure at each offset for the two example subregions. The lowest $\chi^{2}$ value $\left(\chi_{0}\right)$ is determined from a parabolic fit to a set of values (shown in black; shown in blue online) near the distribution minimum. The $850 \mu \mathrm{m}$ offsets corresponding with these minima are given in the bottom right corners along with the observed emission at $850 \mu \mathrm{m}\left(S_{850}\right)$.

(A color version of this figure is available in the online journal.)

line transitions are generally negligible compared to the dust emission. For example, the dust emission rises toward shorter wavelengths as $v^{\beta+2}$, whereas the emission from higher transitions of $\mathrm{CO}$ does not rise as steeply. For example, we do not believe that the excess brightness at $450 \mu \mathrm{m}$ relative to the filtered $500 \mu \mathrm{m}$ emission (see Figures 8 and 11) is due to CO (6-5) contamination at $450 \mu \mathrm{m}$. Since the CO (3-2) line contamination is relatively minor, we can also assume that the contamination from less abundant molecules (i.e., ${ }^{13} \mathrm{CO}$ or $\mathrm{C}^{18} \mathrm{O}$ ) should be negligible.

\section{APPENDIX C}

\section{HERSCHEL COLOR CORRECTIONS}

The PACS and SPIRE fluxes from HIPE ${ }^{26}$ contain several sources of uncertainty. First, both instruments have a $10 \%$ flux error based on the uncertainties in the calibration. For PACS, flux calibrations are mainly based on stellar sources and bright asteroids (Poglitsch et al. 2010), whereas for SPIRE, flux calibrations primarily use Neptune (Griffin et al. 2010). Second,

26 The Herschel Interactive Processing Environment (HIPE) is a joint development software by the Herschel Science Ground Segment Consortium, consisting of ESA, the NASA Herschel Science Center, and the HIFI, PACS, and SPIRE consortia. both PACS and SPIRE calibrations assume that the observed spectral profile follows a $v^{-1}$ power law such that $v S_{v}$ is flat (Poglitsch et al. 2010; Griffin et al. 2010). For cold molecular clouds $(T \sim 10 \mathrm{~K})$, emission in the PACS and SPIRE bands will not follow a $v^{-1}$ power law, and thus color corrections are necessary (e.g., see Pezzuto et al. 2012). Furthermore, the spectral color corrections are not systematic, and they depend on the SED shape and thus will vary with frequency, temperature, and opacity.

Calculating the spectral color corrections is nontrivial. First, these correction factors require foreknowledge of the SED profiles. Second, the color corrections compare the flux from the true SED profile to the assumed SED profile, both weighted by the filter response function. For SPIRE, the beam solid angle varies with wavelength, resulting in more power at longer wavelengths. While this effect is insignificant for point sources, extended sources (i.e., objects that uniformly fill the entire beam) will be biased toward longer wavelengths. Thus, color corrections vary between point sources and extended objects. ${ }^{27}$ PACS calibration does not differentiate between point sources

\footnotetext{
27 For additional information, see the SPIRE Observers' Manual (2011), HERSCHEL-DOC-0798, ver. 2.4, http://herschel.esac.esa.int/Docs/SPIRE/pdf/spire_om.pdf.
} 

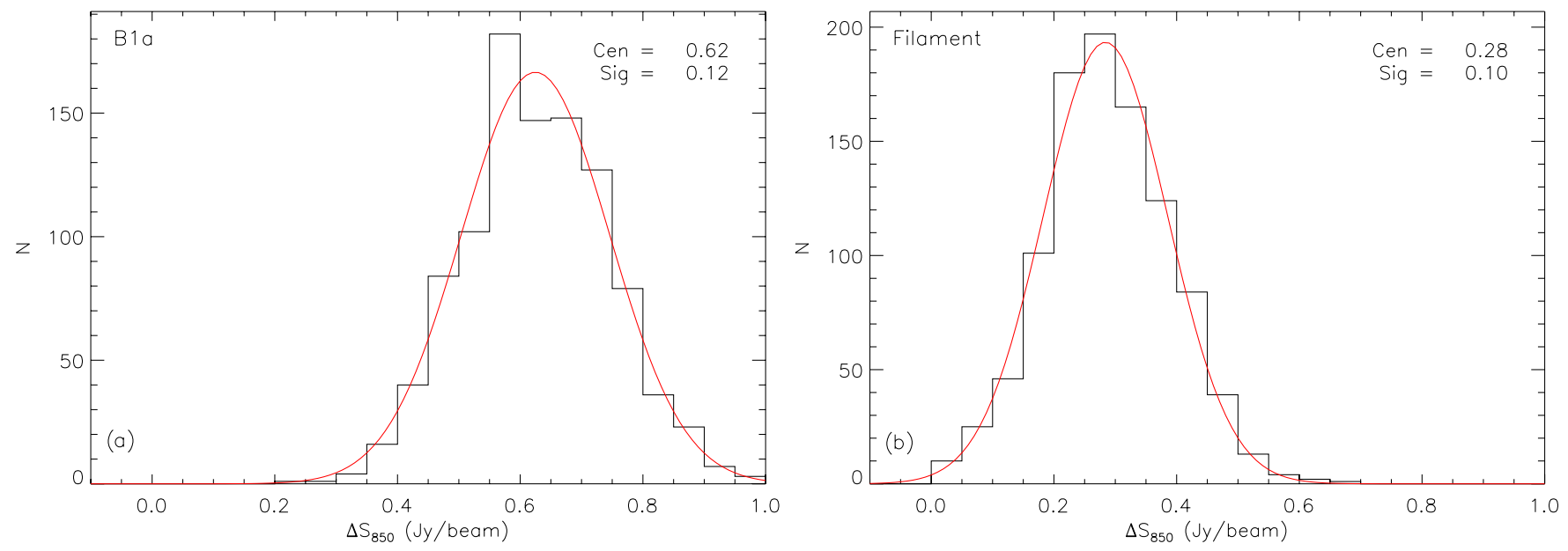

Figure 16. Range of best-fit $850 \mu \mathrm{m}$ offsets. We generated 1000 random calibration correction factors as described in Section 4 , and then found the best-fit $850 \mu \mathrm{m}$ offset for each set of correction factors according to our technique (see the text and Figure 15). The gray curves (red curves online) show a Gaussian fit to the histograms.

(A color version of this figure is available in the online journal.)

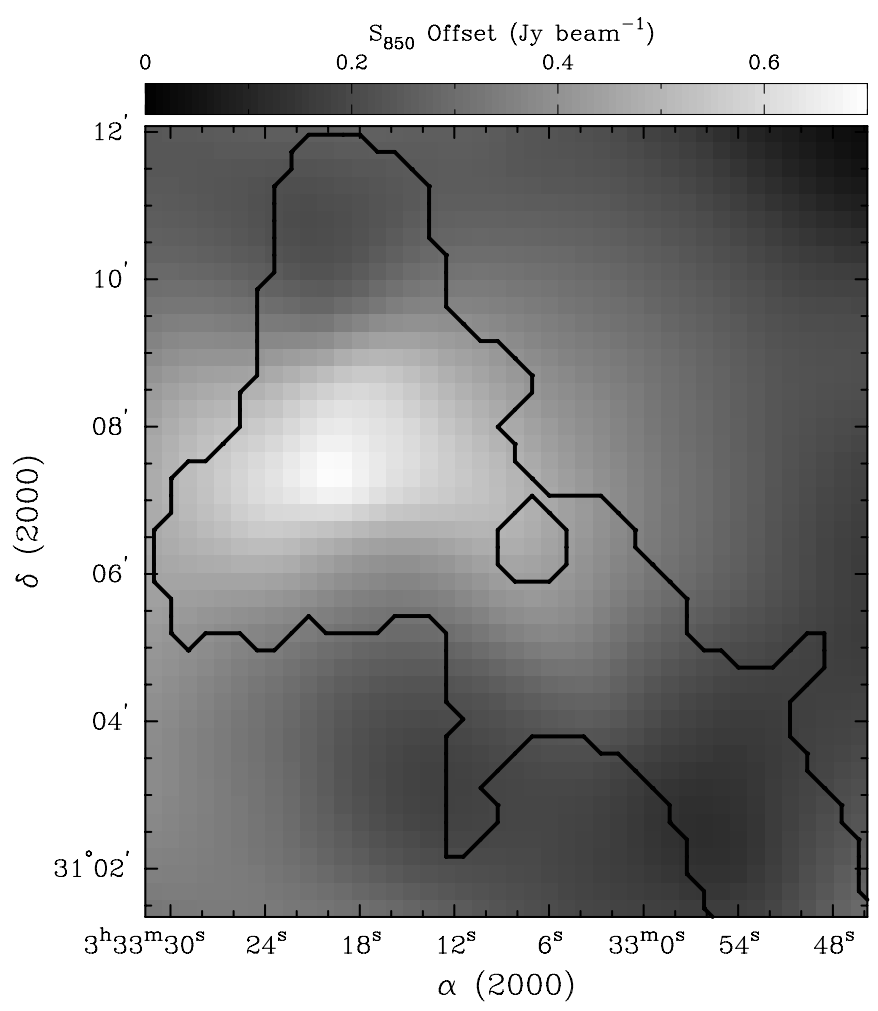

Figure 17. Recovered diffuse emission at $850 \mu \mathrm{m}$ obtained with our technique. The black contours correspond to the $10 \sigma$ region in Section 4.1 .

and extended sources. Since we are interested in the extended Herschel map structures, we will adopt the extended color corrections for SPIRE. We caution that these structures likely do not fill the filter beam uniformly at all wavelengths, and that these color corrections represent our best estimate. For SPIRE, these extended source corrections are generally minor (few percent) and should not greatly affect our results (Griffin et al. 2010). For PACS, however, the color corrections of cold objects can be more significant, particularly at $70 \mu \mathrm{m}$ (Poglitsch et al. 2010). We do not include $70 \mu \mathrm{m}$ emission in the SED fits in this paper, however.

Since the SED profiles are unknown a priori, we found the color corrections associated with cold dust emission at temperatures between $10 \mathrm{~K}$ and $15 \mathrm{~K}$ and dust emissivity indices between 1.5 and 2.5. For PACS, the color corrections for cold and dusty environments are presented on the PACS calibration website by Müller et al. (2011), ${ }^{28}$ and we extrapolated those tabulated values to identify the color corrections associated with our adopted ranges of temperature and $\beta$. For SPIRE, we calculated the color corrections by integrating model SEDs weighted by the relative spectral response function (RSRF) ${ }^{29}$ of each band (e.g., see Pezzuto et al. 2012). We scaled the RSRF profiles such that an SED with a $v^{-1}$ profile would yield color corrections of 1.0 (i.e., what was initially assumed in the calibration). Assuming various power-law profiles, our color corrections agree with the corrections in the SPIRE Observers' Manual to within a few percent. For both PACS and SPIRE, we adopted the midpoint between the maximum and minimum color correction, taking the difference of the average value with respect to the range of accepted values as our uncertainty. Table 1 lists our adopted color corrections for each wavelength. We caution that these values are only valid for $T \approx 10-15 \mathrm{~K}$ and $\beta \approx 1.5-2.5$.

\section{APPENDIX D}

\section{MEASURING $\beta$ FROM UNFILTERED MAPS}

In this Appendix, we outline the techniques for measuring $\beta$ while recovering the missing large-scale structure in the SCUBA-2 maps. These techniques are the Offset Case (Appendix D.1) and the Spatial Factor Case (Appendix D.2). We highlight the method and discuss the uncertainties in the determined values of $\beta$.

\section{D.1. Offset Case}

We use the Herschel data to recover the large-scale structure at $850 \mu \mathrm{m}$. We assume that the missing large-scale emission corresponds to scales $>2.5$, and thus we do not expect large

\footnotetext{
28 PACS Photometer Passbands and Color Correction Factors for Various Source SEDs, PICC-ME-TN-038, ver. 1.0, http://herschel.esac.esa.int/ twiki/pub/Public/PacsCalibrationWeb/cc_report_v1.pdf.

${ }^{29}$ For extended emission, the RSRF profile is multiplied by $\lambda^{2}$ to account for the differences in the beam size between the short-wavelength end and the long-wavelength end of the filter. For more details, see the SPIRE Observers' Manual.
} 

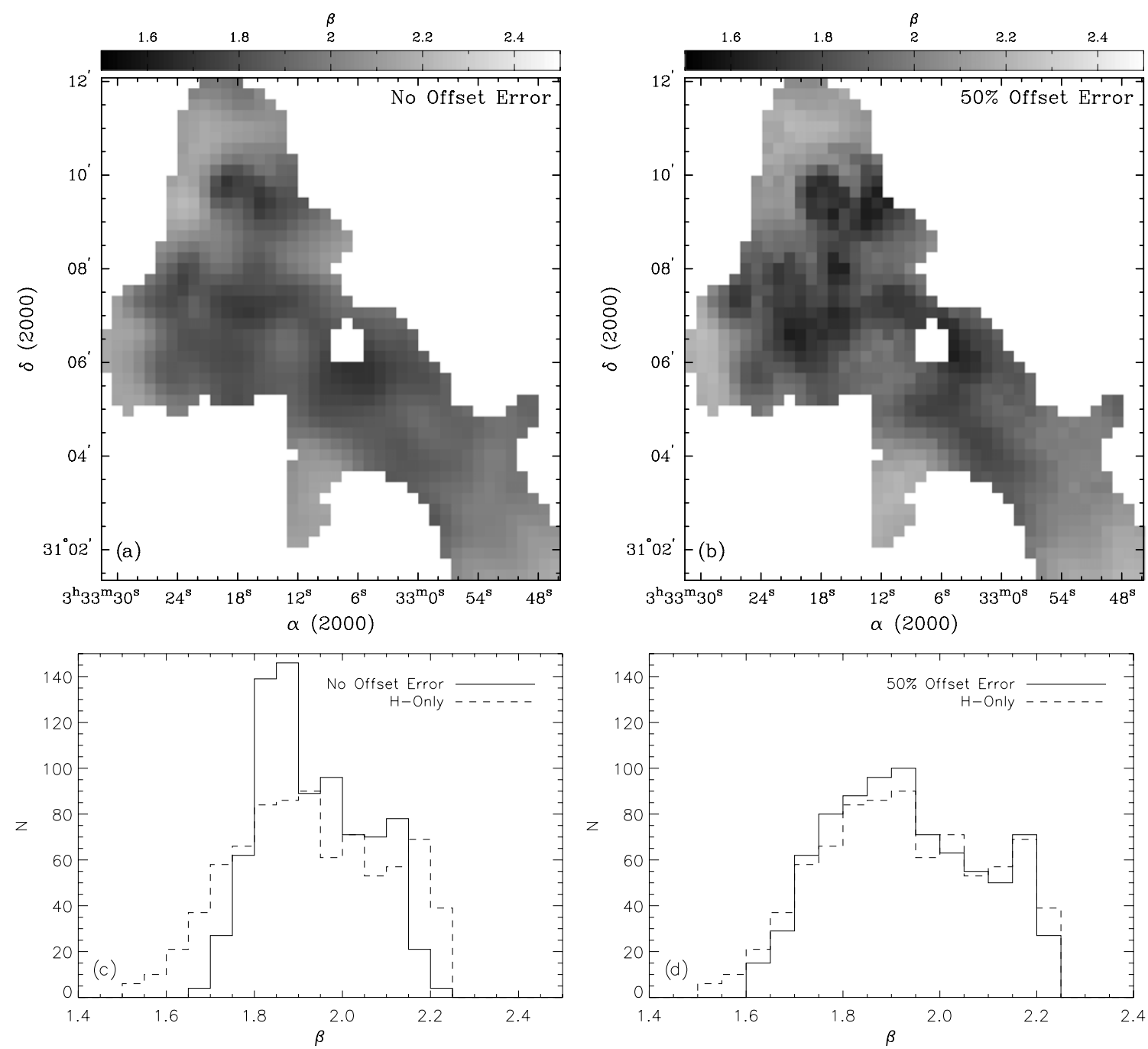

Figure 18. Top: dust emissivity maps assuming (a) no errors for the $850 \mu \mathrm{m}$ offsets or (b) $50 \%$ errors for the $850 \mu \mathrm{m}$ offsets. Bottom: histograms comparing $\beta$ distributions from the $850 \mu \mathrm{m}$ offset analyses and the distribution obtained from H-only data. We assume no $850 \mu \mathrm{m}$ offset uncertainties in (c) and $50 \%$ offset uncertainties in (d).

variations in the extended structure for scales $<2.5$. The method is outlined below and in Figure 15.

1. For each pixel, we select a $9 \times 9$ pixel subregion (corresponding to $\sim 2$ '. 1 for $14^{\prime \prime}$ pixels). The extended emission should not vary over this subregion. See the top panels of Figure 15.

2. We create a grid of $850 \mu \mathrm{m}$ flux offsets ranging from $0 \mathrm{Jy} \mathrm{beam}{ }^{-1}$ to $1.5 \mathrm{Jy} \mathrm{beam}^{-1}$ in increments of $0.03 \mathrm{Jy} \mathrm{beam}^{-1}$ to represent the filtered-out emission at $850 \mu \mathrm{m}$

3. For each $850 \mu \mathrm{m}$ offset, we fit the $160-850 \mu \mathrm{m}$ emission from all pixels within the $9 \times 9$ pixel subregion.

4. We total the individual $\chi^{2}$ measures across the entire subregion for each estimation of the $850 \mu \mathrm{m}$ offset. If the $850 \mu \mathrm{m}$ offset is close to the filtered-out extended emission, then the total $\chi^{2}$ will be low, and if the $850 \mu \mathrm{m}$ offset differs from the true extended emission, then the total $\chi^{2}$ will be high.

5. For the central pixel in the subregion, we adopt the $850 \mu \mathrm{m}$ offset value that corresponds to the minimum total $\chi^{2}$. For simplicity, we fit a parabolic function to the distribution of the total $\chi^{2}$ with offset. See the bottom panels of Figure 15.
Figure 15 illustrates our technique. The top panels show two example subregions with $9 \times 9$ pixel boxes used to measure the offset at the marked positions toward a pixel in B1-a and the southwest filament. For each estimate of the $850 \mu \mathrm{m}$ offset ranging from $0 \mathrm{Jy} \mathrm{beam}^{-1}$ to $1.5 \mathrm{Jy}_{\text {beam }}^{-1}$, we found the best-fit SEDs across these subregions, and then summed the $\chi^{2}$ values from all 81 pixels. The bottom panels show the distributions of total $\chi^{2}$ with $850 \mu \mathrm{m}$ offsets for our two example subregions. As expected, when the $850 \mu \mathrm{m}$ offset is greatly underestimated or overestimated, the total $\chi^{2}$ is large. We used parabolic fits to identify the offset corresponding to the minima. For simplicity, Figure 15 does not include the $10 \%$ calibration uncertainties for each instrument.

Figure 16 shows the range of best-fit $850 \mu \mathrm{m}$ offsets within the $10 \%$ calibration uncertainties for the two pixels highlighted in Figure 15. The range of acceptable offsets appears fairly broad, suggesting that this technique does not well constrain the missing $850 \mu \mathrm{m}$ large-scale emission and these uncertainties overwhelm the uncertainties of the observed $850 \mu \mathrm{m}$ data (10\%). Applying 1000 random calibration correction factors to each subregion (effectively 81 SEDs per increment) and each estimated $850 \mu \mathrm{m}$ offset (50 possible increments) is computationally expensive. Therefore, we sampled a subset of 10 pixels and found that the $1 \sigma$ standard deviation 

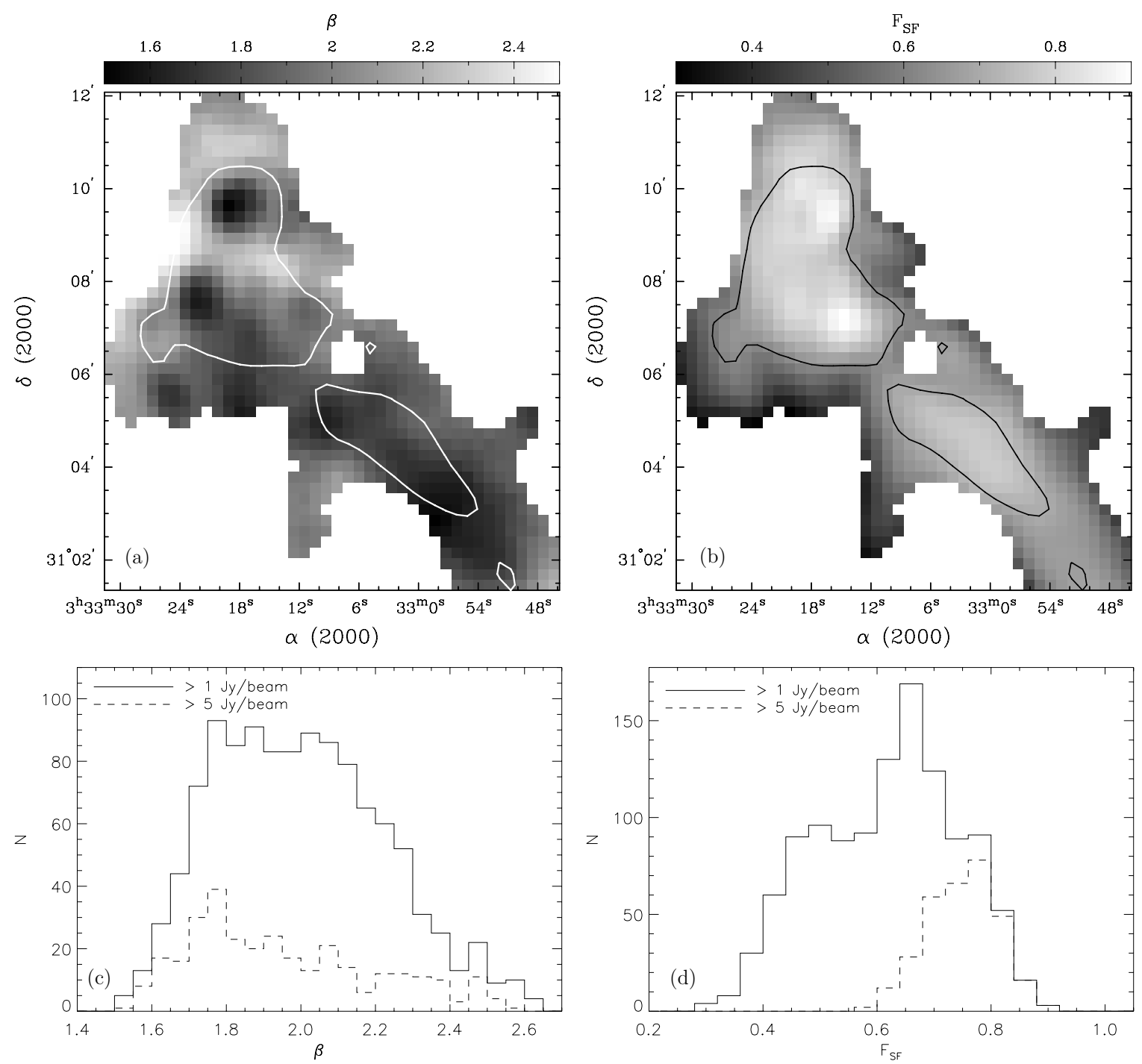

Figure 19. Top: results from the Spatial Factor Case highlighting (a) the dust emissivity and (b) spatial filtering factor $\left(F_{\mathrm{SF}}\right)$. The dust emission was fit using Equation (3), assuming an equal scaling factor, $F_{\mathrm{SF}}$ for the $450 \mu \mathrm{m}$ band and the $850 \mu \mathrm{m}$ band. The contours indicate the region with $I_{450}>5 \mathrm{Jy}_{\text {beam }}{ }^{-1}$. Bottom: histograms for $\beta$ and spatial filtering factor. The solid lines show the histograms for the entire region in the upper panels, and the dashed lines show the histograms for the $I_{450}>5 \mathrm{Jy}_{\text {beam }}{ }^{-1}$ regions, only. For these analyses, the $450 \mu \mathrm{m}$ data were scaled downward by $12.5 \%$ to account for the fractional increase in brightness in the observed maps over the predicted maps (see Figure 11).

errors in the mean offsets are $20 \%-50 \%$ of the respective offset measures.

Figure 17 shows the "recovered" $850 \mu \mathrm{m}$ large-scale emission. The black contours show the $10 \sigma$ flux level from Section 4.1. The recovered $850 \mu \mathrm{m}$ extended emission shows some similarities and yet some variations with the filtered-out large-scale emission at $250 \mu \mathrm{m}$ shown in Figure 6. For example, we see the recovered $850 \mu \mathrm{m}$ large-scale emission peak toward the central B1 region at both wavelengths. Conversely, the recovered $850 \mu \mathrm{m}$ large-scale emission map appears more structured, with less extended emission toward the main filament and B1-c. Since the $850 \mu \mathrm{m}$ emission traces very cold dust, these differences could reflect differences in dust temperature. Similar differences, however, are not seen between the Herschel bands at $250 \mu \mathrm{m}$ and $500 \mu \mathrm{m}$.

Figure 18 shows the $\beta$ maps determined from SED fitting with the Herschel +850 bands, where the $850 \mu \mathrm{m}$ emission includes the recovered extended structure from Figure 17. We measured $\beta$ assuming two extremes, i.e., (1) no uncertainties in the extended emission and (2) 50\% uncertainties in the extended emission.
In the bottom panels, we compare the derived $\beta$ distributions with the equivalent $\mathrm{H}$-only distribution. Most noticeably, there is a spike at $\beta \approx 1.8-1.9$ in Figure 18(c), which is similar to the $\beta \approx 2$ peak seen in Figure 9. Nevertheless, as shown in Figure 18(d), this spike disappears if we assume 50\% errors for the $850 \mu \mathrm{m}$ extended emission, likely due to the $850 \mu \mathrm{m}$ data being too uncertain to constrain well the SEDs. Indeed, the $\beta$ distribution with $50 \%$ flux errors in the $850 \mu \mathrm{m}$ extended structure appears more similar to the H-only distribution, with a slight offset toward lower $\beta$ values. Therefore, the Offset Case approach to recover the filtered-out, large-scale $850 \mu \mathrm{m}$ emission makes the $850 \mu \mathrm{m}$ data too uncertain to be an effective constraint.

\section{D.2. Spatial Factor Case}

For the Spatial Factor Case, we attempt to recover the missing large-scale emission by scaling the $450 \mu \mathrm{m}$ and $850 \mu \mathrm{m}$ emission by a spatial filtering factor to account for the missing extended flux. We assume that the $450 \mu \mathrm{m}$ and $850 \mu \mathrm{m}$ observations are missing the same fraction of extended emission, and 
then fit the SEDs according to Equation (3), solving for the SED parameters and the spatial filtering factor, $F_{\mathrm{SF}}$. Since Figure 11 demonstrates that the observed $450 \mu \mathrm{m}$ emission tends to be $10 \%-15 \%$ brighter than the predicted emission, we adjusted the $450 \mu \mathrm{m}$ emission downward by $12.5 \%$. This adjustment appears most reasonable for the brightest parts of the B1 clump (i.e., $I_{450}>5 \mathrm{Jy} \mathrm{beam}^{-1}$ ), however, and may cause erroneous results outside of these locations.

The top panels of Figure 19 show the $\beta$ values and spatial filtering factors derived using Equation (3). The contours correspond to the regions with $I_{450}>5 \mathrm{Jy}_{\text {beam }}{ }^{-1}$, and where a scaling of $-12.5 \%$ is more reasonable (see Figure 11 ). Similar to the previous measures of $\beta$, we find that $\beta$ is lowest toward B1-c. On the other hand, we find $\beta \sim 2.3$ toward B1-a and $\beta \sim 1.6$ toward B1-b. Additionally, we obtain $\beta \sim 1.7$ along the filament. For the spatial filtering factor, we find $F_{\mathrm{SF}} \sim 0.8-0.9$ toward the dense, compact cores and $F_{\mathrm{SF}} \sim 0.7$ toward the filament. Toward the edges of the $10 \sigma$ region, however, the spatial filtering factor drops to $\lesssim 0.4$, suggesting that most of that emission was removed by the SCUBA-2 reduction pipeline. Figure 19 also shows the distributions of $\beta$ and $F_{\mathrm{SF}}$ for the $>1 \mathrm{Jy} \mathrm{beam}^{-1}$ and $>5 \mathrm{Jy} \mathrm{beam}^{-1}$ regions. Unsurprisingly, the brightest regions (i.e., the locations of the compact objects) correspond with the highest fraction of recovered emission. Nevertheless, we find a wide range of $\beta$ values even for the $>5 \mathrm{Jy} \mathrm{beam}^{-1}$ region. Within the calibration uncertainties, we find $\sigma_{\beta} \lesssim 1.0$. Therefore, the Spatial Filtering Case is not a robust method to determine $\beta$.

\section{REFERENCES}

André, P., Men'shchikov, A., Bontemps, S., et al. 2010, A\&A, 518, L102

Arab, H., Abergel, A., Habart, E., et al. 2012, A\&A, 541, A19

Arzoumanian, D., André, P., Didelon, P., et al. 2011, A\&A, 529, L6

Bally, J., Walawender, J., Johnstone, D., Kirk, H., \& Goodman, A. 2008, in Handbook of Star Forming Regions, Vol 1, ed. B. Reipurth (San Francisco, CA: ASP), 308

Beckwith, S. V. W., \& Sargent, A. I. 1991, ApJ, 381, 250

Bergin, E. A., \& Tafalla, M. 2007, ARA\&A, 45, 339

Bernard, J.-P., Paradis, D., Marshall, D. J., et al. 2010, A\&A, 518, L88

Buckle, J. V., Hills, R. E., Smith, H., et al. 2009, MNRAS, 399, 1026

Cavanagh, B., Jenness, T., Economou, F., \& Currie, M. J. 2008, AN, 329, 295

Chapin, E. L., Berry, D. S., Gibb, A. G., et al. 2013, MNRAS, in press (arXiv:1301.3652)

Davis, C. J., Dent, W. R. F., Matthews, H. E., Coulson, I. M., \& McCaughrean, M. J. 2000, MNRAS, 318, 952

Dempsey, J. T., Friberg, P., Jenness, T., et al. 2013, MNRAS, in press (arXiv:1301.3773)

Dempsey, J. T., Holland, W. S., Chrysostomou, A., et al. 2012, Proc. SPIE, 8452,845202

Di Francesco, J., Johnstone, D., Kirk, H., MacKenzie, T., \& Ledwosinska, E. 2008, ApJS, 175, 277

Doty, S. D., \& Leung, C. M. 1994, ApJ, 424, 729

Drabek, E., Hatchell, J., Friberg, P., et al. 2012, MNRAS, 426, 23

Draine, B. T., \& Lee, H. M. 1984, ApJ, 285, 89

Dupac, X., Bernard, J.-P., Boudet, N., et al. 2003, A\&A, 404, L11
Enoch, M. L., Evans, N. J., II, Sargent, A. I., \& Glenn, J. 2009, ApJ, 692, 973

Friesen, R. K., Johnstone, D., Naylor, D. A., \& Davis, G. R. 2005, MNRAS, 361,460

Griffin, M. J., Abergel, A., Abreu, A., et al. 2010, A\&A, 518, L3

Hatchell, J., Fuller, G. A., Richer, J. S., Harries, T. J., \& Ladd, E. F. 2007, A\&A, 468, 1009

Hatchell, J., Wilson, T., Drabek, E., et al. 2013, MNRAS, 429, L10

Henning, T., Michel, B., \& Stognienko, R. 1995, P\&SS, 43, 1333

Hildebrand, R. H. 1983, QJRAS, 24, 267

Hill, T., Thompson, M. A., Burton, M. G., et al. 2006, MNRAS, 368, 1223

Hirota, T., Bushimata, T., Choi, Y. K., et al. 2008, PASJ, 60, 37

Holland, W. S., Bintley, D., Chapin, E. L., et al. 2013, MNRAS, in press (arXiv:1301.3650)

Holland, W. S., Robson, E. I., Gear, W. K., et al. 1999, MNRAS, 303, 659

Jenness, T., Berry, D., Chapin, E., et al. 2011, in ASP Conf. Ser. 442, Astronomical Data Analysis Software and Systems XX, ed. I. N. Evans, A. Accomazzi, D. J. Mink, \& A. H. Rots (San Francisco, CA: ASP), 281

Jenness, T., Cavanagh, B., Economou, F., \& Berry, D. S. 2008, in ASP Conf. Ser. 394, Astronomical Data Analysis Software and Systems XVII, ed. R. W. Argyle, P. S. Bunclark, \& J. R. Lewis (San Francisco, CA: ASP), 565

Johnstone, D., Boonman, A. M. S., \& van Dishoeck, E. F. 2003, A\&A, 412, 157

Jørgensen, J. K., Johnstone, D., Kirk, H., \& Myers, P. C. 2007, ApJ, 656, 293

Jørgensen, J. K., Johnstone, D., Kirk, H., et al. 2008, ApJ, 683, 822

Kauffmann, J., Bertoldi, F., Bourke, T. L., Evans, N. J., II, \& Lee, C. W. 2008, A\&A, 487, 993

Kelly, B. C., Shetty, R., Stutz, A. M., et al. 2012, ApJ, 752, 55

Kirk, H., Johnstone, D., \& Di Francesco, J. 2006, ApJ, 646, 1009

Lis, D. C., Serabyn, E., Keene, J., et al. 1998, ApJ, 509, 299

Markwardt, C. B. 2009, in ASP Conf. Ser. 411, Astronomical Data Analysis Software and Systems XVIII, ed. D. A. Bohlender, D. Durand, \& P. Dowler (San Francisco, CA: ASP), 251

Martin, P. G., Roy, A., Bontemps, S., et al. 2012, ApJ, 751, 28

Matthews, B. C., Hogerheijde, M. R., Jørgensen, J. K., \& Bergin, E. A. 2006, ApJ, 652, 1374

Meny, C., Gromov, V., Boudet, N., et al. 2007, A\&A, 468, 171

Ossenkopf, V., \& Henning, T. 1994, A\&A, 291, 943

Paradis, D., Veneziani, M., Noriega-Crespo, A., et al. 2010, A\&A, 520, L8

Pezzuto, S., Elia, D., Schisano, E., et al. 2012, A\&A, 547, A54

Pilbratt, G. L., Riedinger, J. R., Passvogel, T., et al. 2010, A\&A, 518, L1

Planck Collaboration, Abergel, A., Ade, P. A. R., Aghanim, N., et al. 2011, A\&A, 536, A25

Poglitsch, A., Waelkens, C., Geis, N., et al. 2010, A\&A, 518, L2

Román-Zúñiga, C. G., Alves, J. F., Lada, C. J., \& Lombardi, M. 2010, ApJ, 725,2232

Rosolowsky, E. W., Pineda, J. E., Foster, J. B., et al. 2008, ApJS, 175, 509

Roussel, H. 2012, submitted (arXiv:1205.2576)

Roy, A., Martin, P. G., Polychroni, D., et al. 2013, ApJ, 763, 55

Sadavoy, S. I., Di Francesco, J., André, P., et al. 2012, A\&A, 540, A10

Schnee, S., Enoch, M., Noriega-Crespo, A., et al. 2010, ApJ, 708, 127

Schwartz, P. R. 1982, ApJ, 252, 589

Shetty, R., Kauffmann, J., Schnee, S., \& Goodman, A. A. 2009a, ApJ, 696, 676

Shetty, R., Kauffmann, J., Schnee, S., Goodman, A. A., \& Ercolano, B. 2009b, ApJ, 696, 2234

Shirley, Y. L., Huard, T. L., Pontoppidan, K. M., et al. 2011, ApJ, 728, 143

Sun, K., Kramer, C., Ossenkopf, V., et al. 2006, A\&A, 451, 539

Tothill, N. F. H., White, G. J., Matthews, H. E., et al. 2002, ApJ, 580, 285

Veneziani, M., Ade, P. A. R., Bock, J. J., et al. 2010, ApJ, 713, 959

Ward-Thompson, D., Di Francesco, J., Hatchell, J., et al. 2007, PASP, 119,855

White, G. J., Araki, M., Greaves, J. S., Ohishi, M., \& Higginbottom, N. S. 2003, A\&A, 407, 589 